\title{
THE FLYING RADIATION CASE
}

\author{
J.H. Brownell and R.L. Bowers
}

Applied Theoretical and Computational Physics Division

Los Alamos National Laboratory, Los Alamos N.M. 87545

\begin{abstract}
The Los Alamos foil implosion program has the goal of producing an intense, high-energy density $\mathrm{x}$-ray source by converting the energy of a magnetically imploded plasma into radiation and material energy. One of the methods for converting the plasma energy into thermal energy and radiation and utilizing it for experiments is called the "flying radiation case (FRC)." In this paper we shall model the FRC and provide a physical description of the processes involved. An analytic model of a planar FRC in the hydrodynamic approximation is used to describe the assembly and shock heating of a central cushion by a conducting liner driver. The results are also used to benchmark a hydrodynamics code for modeling an FRC. We then use a radiation-hydrodynamics computational model to explore the effects of radiation production and transport when a gold plasma assembles on a $\mathrm{CH}$ cushion. Results are presented for the structure and evolution of the radiation hohlraum.

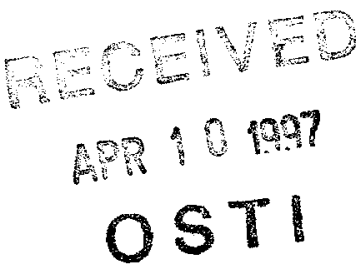

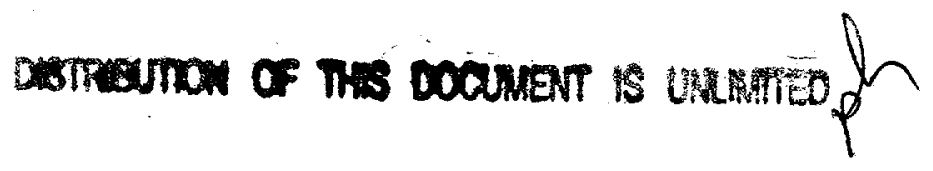

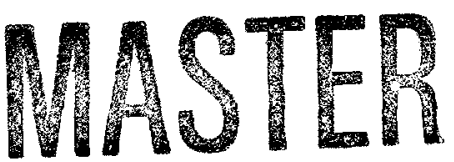




\section{DISCLAIMER}

This report was prepared as an account of work sponsored by an agency of the United States Government. Neither the United States Government nor any agency thereof, nor any of their employees, make any warranty, express or implied, or assumes any legal liability or responsibility for the accuracy, completeness, or usefulness of any information, apparatus, product, or process disclosed, or represents that its use would not infringe privately owned rights. Reference herein to any specific commercial product, process, or service by trade name, trademark, manufacturer, or otherwise does not necessarily constitute or imply its endorsement, recommendation, or favoring by the United States Government or any agency thereof. The views and opinions of authors expressed herein do not necessarily state or reflect those of the United States Government or any agency thereof. 


\section{DISCLAMMER}

Portions of this document may be illegible in electronic image products. Images are produced from the best available original document. 


\section{INTRODUCTION}

The Los Alamos foil implosion program has the goal of producing an intense, high-energy density $\mathrm{x}$-ray source by converting the energy of a magnetically imploded plasma into radiation and material internal energy. The intermediate-term goal is to obtain a source with which experiments on radiation transport, opacity, equation of state, and implosion physics may be performed. Ultimately, with a multi-megajoule source it is envisioned that fusion experiments will be possible.

At Los Alamos one of the methods for converting the kinetic energy of the plasma into thermal energy and radiation and utilizing it for experiments has been dubbed the "flying radiation case" (FRC). This is basically a cylindrical driver plasma which implodes and stagnates upon a centrally-located cushion. It is arranged for the driver to become optically thick at the time of maximum convergence in order to inhibit the radiative cooling of the cushion. Figure 1 shows several representative geometries which utilize the FRC for physics experiments in cylindrical geometry.

The backside of the driver-plasma is magnetically Rayleigh-Taylor unstable and perturbations which are present in the initial foil, or which arise during the implosion, will grow ${ }^{1}$. If the perturbations are of sufficient amplitude, or if the ratio of plasma shell thickness to run-in distance is small, the instabilities may disrupt the driver. Physical arguments and two-dimensional (2-D) magnetohydrodynamic (MHD) calculations indicate that an imploding driver plasma will tend to reassemble when it impacts a cushion material. Thus, the cushion can lead to a more nearly homogeneous central radiation environment than would be obtained from the implosion of a simple plasma which stagnates on axis. Two-dimensional models of these effects are necessary in arriving at reasonable estimates of temperatures and the radiation output from a FRC. Consistent modeling of an FRC implosion should include the coupling of the pulsed power system to the

\footnotetext{
${ }^{1}$ R.L. Bowers, J.H. Brownell, A.E. Greene, H. Kruse, H. Oona, D.L. Peterson, "Nonlinear Interaction of Magnetohydrodynamic Instabilities in Radially Imploded Plasmas", Megagauss Magnetic Field Generation and PulsedPower Applications, ed. by. M. Cowan and R.B. Spielman, (Nova Science Publishers, Inc: New York, 1994) pg. 777; and D.L. Peterson, R.L. Bowers, J.H. Brownell, A.E. Greene, H.W. Kruse, H. Lee and W. Matuska, "Radiation Magnetohydrodynamic Modeling of X-Ray Output from Perturbed Plasma Implosions", Megagauss Magnetic Field Generation and PulsedPower Applications, ed. by. M. Cowan and R.B. Spielman, (Nova Science Publishers, Inc: New York, 1994) pg. 897.
} 
gamma $\gamma=\gamma_{C}$, which is initially at rest against a solid, impenetrable wall (Figure 2). We choose time $t=0$ as the instant when the driver plasma impacts the cushion. When the driver impacts the . cushion, shocks are generated in both media (Figure 3). The shock in the cushion travels inward toward the reflecting plane in our model until it contacts the wall where it is reflected at time $\mathrm{t}_{1}$ (Figure 3). It then moves outward reaching the contact discontinuity at time $t 2$. The shock in the driver moves outward until it reaches the outer boundary, where a rarefaction forms and travels back towards the contact discontinuity .

The driver consists of a relatively low density plasma (typical densities are expected to be in the range of $10^{-4}$ to $10^{-5} \mathrm{~g} / \mathrm{cm}^{3}$ ) which, because of Joule heating and radiative losses will be at temperatures of $5-50 \mathrm{eV}$. Prior to impact $(\mathrm{t}=0)$, the cushion will be preheated by radiation from this hot plasma. Shock heating, associated with impact between the driver and the cushion, also produces a radiation front which moves into the cushion supersonically for cushion materials of interest here. In the next section the hydrodynamic evolution is followed from first impact to maximum compression of the cushion. In Section IV we include the radiation front structure. One of the goals is to find the material and radiation temperature history in the cushion. The detailed behavior depends on the fluid variables and initial dimensions, and we shall find the parameters that optimize the hohlraum conditions.

The problem will be described by means of the radiation-hydrodynamic equations. In the energy range of interest for our applications the radiation pressure and radiation energy density are ignorable, but it is necessary to include the radiation flux in the equations. Similarity solutions to the remaining equations are well-known ${ }^{3}$, but they break down in the presence of a symmetry axis, or an external boundary (such as the impenetrable wall assumed in our model). We consider the evolution of the system in two steps. First, from the hydrodynamic equations we obtain the plasma temperature resulting from shock heating of the materials. In the case of an optically thick cushion, the radiation temperature and the plasma temperatures will be comparable. In the case of

\footnotetext{
${ }^{3}$ I.P. Raizer, Soviet Physics JETP, 5, No.6, 1957, pg. 1242; M.A. Heaslet and B. S. Baldwin, Phys. Fluids, 6, No. 6, 1963, pg. 781; M. Mitchner and M. Vinokur, Phys. Fluids, 6, No. 12, 1963, pg. 1682.
} 
an optically thin cushion, a numerical model will be solved to give the hohlraum temperature as a function of the velocity and temperature of the driver plasma.

To describe the flow before the fronts encounter boundaries, Hugoniot relations including radiative flux will be applied. For our application it is more convenient to express these relations in the laboratory frame (see the Appendix). In the Appendix we also show that away from the front the flow variables approach those that would be derived in the absence of radiative effects. These flow variables will be found as functions of the initial driver and cushion conditions, and from this a good understanding of the energy conversion dynamics will be gained.

\section{HYDRODYNAMICS OF THE ASSEMBLY}

We now consider the hydrodynamic evolution of the driver-cushion assembly. An analysis of the time development will be done in two stages, shown schematically in Figure 3, which correspond to the time intervals: $0<\mathrm{t}<\mathrm{t}_{1}$ (direct shock heating of the cushion); and $\mathrm{t}_{1}<\mathrm{t}<\mathrm{t}_{2}$ (heating of the cushion by the reflected shock); the interval $t>t_{2}$ (stagnation and beginning of disassembly) will not be considered here. Including the effects of the MHD drive will produce late time tamping of the FRC, and will change the results.

\section{A. Shock Heating $\left(0<t<t_{1}\right)$}

Consider the driver plasma with initial density, $\rho F o$, and pressure, $P_{F o}$, that is initially in motion with velocity, $v_{O}$, and which collides with the cushion at rest with initial density, $\rho \mathrm{Co}_{0}$, and pressure, $\mathrm{PCo}$. Let us find the solution for the flow variables for the case where radiation is ignored. By the proof given in the Appendix this will give the exact solution at points removed from the front. The subsequent motion is described by Figure 3, which shows the space-time diagram of the dynamics (the subscript 1 denotes the shocked state). The relations that describe the flow from the time of impact to the time that the shocks reach the outside boundary of the driver plasma (denoted by subscript $F$ ) and/or the cushion (denoted by subscript $C$ ) are:

\section{Driver:}




$$
\begin{gathered}
\rho_{F o}\left(U_{F}+v_{o}\right)=\rho_{F I}\left(U_{F}+u_{F 1}\right) \\
\rho_{F O}\left(U_{F}+v_{O}\right)\left(u_{F I}-v_{0}\right)=-\left(P_{F I}-P_{F O}\right) \\
\rho_{F O}\left(U_{F}+v_{0}\right)\left(\varepsilon_{F I}-\varepsilon_{F O}+\frac{1}{2} u_{F I}^{2}-\frac{1}{2} v_{\delta}\right)=P_{O} v_{\sigma}-P_{1} u_{F 1},
\end{gathered}
$$

where $U$ denotes the shock velocity and $\varepsilon$ is the specific internal energy.

Cushion:

$$
\begin{gathered}
\rho_{C O} U_{C}=\rho_{C I}\left(U_{C}-u_{C 1}\right) \\
\rho_{C O} U_{C}^{u_{C I}}=P_{C I}-P_{C O} \\
\rho_{C O} U_{C}\left(\varepsilon_{C I}-\varepsilon_{C O}+\frac{1}{2} u_{C I}^{2}\right)=P_{C I} u_{C l}
\end{gathered}
$$

Letting $\rho=1 / V$, where $V$ is the specific volume, it is easily shown that

$$
\begin{aligned}
& U_{F}=\frac{V_{F 0} u_{F 1}}{V_{F 1}-V_{F O}}-\frac{V_{F 1} v_{0}}{V_{F 1}-V_{F O}} \\
& \left(u_{F 1}-v_{0}\right)^{2}=\left(P_{F 1}-P_{F 0}\right)\left(V_{F O}-V_{F 1}\right) \\
& \varepsilon_{F 1}-\varepsilon_{F O}=\frac{l}{2}\left(P_{F 1}+P_{F O}\right)\left(V_{F O}-V_{F 1}\right)
\end{aligned}
$$

and

$$
U_{C}=\frac{V_{C 0} u_{C I}}{V_{C O}-V_{C I}}
$$




$$
\begin{aligned}
& u_{C I}{ }^{2}=\left(P_{C I}-P_{C O}\right)\left(V_{C O}-V_{C I}\right) \\
& \varepsilon_{C I}-\varepsilon_{C O}=\frac{1}{2}\left(P_{C I}+P_{C O}\right)\left(V_{C O}-V_{C I}\right)
\end{aligned}
$$

Using (4b) and (5b) to eliminate the specific volumes and defining the effective heat capacity $\mathrm{CV}$ through the relation

$$
\varepsilon=\frac{P V}{\gamma-I}=C_{V} T
$$

and noting that at the contact discontinuity $\mathrm{u}_{\mathrm{C} 1}=\mathrm{uF}_{\mathrm{F}}=\mathrm{u}$, and $\mathrm{PC1}=\mathrm{PF}_{\mathrm{F}}=\mathrm{P}$, we find in the strong shock limit $\mathrm{P}>>\mathrm{PF} 0$ and $\mathrm{P} \gg \mathrm{PCO}$

$$
u=\frac{v_{0}}{1+\alpha}
$$

where

$$
\alpha \equiv\left\{\frac{\rho_{C O}}{\rho_{F O}} \frac{\gamma_{C}+1}{\gamma_{F}+1}\right\}^{1 / 2}
$$

The plus sign in the denominator has been chosen to satisfy analytic contiuation in the parameters $\rho$ and $\gamma$. In addition, the pressure and velocity in the cushion behind the shock can be written in the form

$$
P=\frac{\gamma_{C}+1}{2} \rho_{C O} u^{2}=\frac{\gamma_{F}+1}{2} \rho_{F O}\left(u-v_{0}\right)^{2}
$$

and

$$
V_{C I}=V_{C 0} \frac{\gamma_{C}-1}{\gamma_{C}+1}
$$

The ratio of the temperature across the shock front in the cushion can be written in the form 


$$
\frac{T_{C l}}{T_{C O}}=\frac{\gamma_{C}-1}{2 P_{C O}} \rho_{C o} \frac{v^{2}}{(1+\alpha)^{2}}
$$

or, equivalently,

$$
T_{C l}=\frac{u^{2}}{2 C_{V}}
$$

It follows from (8) and (11) that the maximum temperature is obtained in the limit $\rho_{\mathrm{C} 0} / \rho_{\mathrm{F} 0} \rightarrow 0$.

Finally, the shock velocities are given by

$$
U_{C}=\frac{\gamma_{C}+1}{2} u
$$

and

$$
U_{F}=\frac{1}{2}\left[\left(\gamma_{F}-1\right) v_{0}-\left(\gamma_{F}+1\right) u\right]
$$

We can now calculate the kinetic and internal energies at the time $t_{1}=\mathrm{d}_{C} / \mathrm{UC}_{\mathrm{C}}\left(\mathrm{d}_{\mathrm{C}}\right.$ is the initial thickness of the cushion) when the shock reaches the far surface of the cushion (Figure 3), assuming that the rarefaction in the driver plasma has not yet reached the contact discontinuity. First, note that the total energy per unit area A (neglecting any initial internal energy) is

$$
\left(E_{0} / A\right)=\frac{1}{2} \rho_{F O} d_{F} v^{2}
$$

Here $\mathrm{dF}_{\mathrm{F}}$ is the initial thickness of the driver plasma. The kinetic energy per unit area of the cushion is found to be

$$
(K E / A)_{C}=\frac{1}{2} \rho_{C O} d_{C} u^{2}=\frac{d_{C} \rho_{C O}}{d_{F} \rho_{F O}} \frac{\left(E_{d} / A\right)}{(1+\alpha)^{2}}
$$

For the internal energy, IE, per unit area of the cushion, we note that in the strong shock limit

$$
(\mathrm{IE} / \mathrm{A}) \mathrm{C}=(\mathrm{KE} / \mathrm{A}) \mathrm{C}
$$

and there is equipartition. For completeness, the driver kinetic energy per unit area is 


$$
\begin{aligned}
& (K E / A)_{F}=\frac{1}{2} \rho_{F O}\left[u t-\left(-d_{F}+v_{0} t\right)-\left(u+\left|U_{F}\right| t\right] v_{\delta}^{2}\right. \\
& +\frac{1}{2} \rho_{F I}\left(u+\left|U_{F}\right\rangle t u^{2}\right.
\end{aligned}
$$

The first term is the kinetic energy of the unshocked plasma, and the second is term is that of the shocked part (it is implicitly assumed at this point that $\mathrm{dF}$ is large enough that the shock has not reached the driver plasma's free surface). After some reduction, (17) becomes

$$
(K E / A)_{F}=\left(E_{d} / A\right)+\frac{\left(E_{d} / A\right)}{d_{F}} \frac{\gamma_{F}+1}{2} \frac{\alpha}{\alpha+1} v_{0^{t}}\left[\left(\frac{1}{1+\alpha}\right)^{2}-1\right]
$$

Finally, it is found that the driver internal energy is

$$
(I E / A)_{F}=\frac{\delta}{2} \rho_{F 1}\left(u-v_{0}\right)^{2}=\frac{\rho_{F I} \delta}{\rho_{F 0^{d}} d_{F}}\left(E_{\delta} / A\right)\left(\frac{\alpha}{1+\alpha}\right)^{2}
$$

where

$$
\delta \equiv \frac{\gamma_{F}-1}{2} v_{0} t \frac{\alpha}{1+\alpha}
$$

At $t_{1}$ a large fraction of the total energy is still in kinetic form, but the material is beginning to heat. For example, we note that in the special case where $d F=d C, \rho F 0=\rho C 0$, and $\gamma_{F}=\gamma C$, there is complete equpartition and $(\mathrm{KE})=(\mathrm{IE})=(\mathrm{E} / \mathrm{A}) / 4$ for both the cushion and the driver plasma.

\section{B. Shock Reflection $\left(t_{1}<t<t_{2}\right)$}

At time $t_{1}$ the shock in the cushion is reflected from the wall, leaving the material behind it at rest. Using the Hugoniot relations (the fluid ahead of the shock is denoted by subscript 1, while the material behind it is denoted by subscript 2 ) it is found that

$$
\frac{P_{2}}{P_{1}}=\frac{3 \gamma_{C}-1}{\gamma_{C}-1}, \quad \frac{\rho_{2}}{\rho_{1}}=\frac{\gamma_{C}}{\gamma_{C}-1}, \frac{T_{2}}{T_{1}}=\frac{3 \gamma_{C}-1}{\gamma_{C}}, \quad U_{C 2}=-\left(\gamma_{C}-1\right) u
$$


where $\mathrm{u}$ is the fluid velocity behind the incident shock. The temperature behind the reflected shock may be expressed in terms of the initial state variables as

$$
T_{C, 2}=\frac{3 \gamma_{C}-1}{2 \gamma_{C}} \frac{u^{2}}{C_{V}}
$$

This is a factor $\left(3 \gamma_{C}-1\right) / \gamma_{C}$ times greater than the temperature before shock reflection and is due to the complete stagnation of the kinetic energy of the cushion. This is the primary reason why the solid cushion has been chosen instead of an annulus. The increased cushion or hohlraum temperature comes at a price, however, in that one must deal with the strong shock on axis which could destroy an experiment that is placed there. In the case of a fusion capsule which is placed in the cushion, for example, the shock can be handled either through placing the capsule inside of a blast shield for protection, or by designing the capsule so that its ablator holds off the shock. That these options are viable has been confirmed with detailed numerical calculations.

\section{Stagnation $\left(t>t_{2}\right)$}

The goal is to convert as much of the internal energy of the driver, $\left(\mathrm{E}_{0} / \mathrm{A}\right)$, as possible into internal energy of the cushion at the time the reflected shock reaches the contact discontinuity ${ }^{4}$. To do this, the kinetic energy of the cushion must be stagnated. If the initial fluid parameters are chosen so that the rarefaction in the driver that travels back toward the cushion does not reach the contact discontinuity before time $t 2$, then the outward moving shock completely stagnates the cushion. The condition, then, to obtained complete stagnation of the cushion is that the position of the rarefaction in the driver at $t_{2}, x_{R}\left(t_{2}\right)$, be to the left (see Figure 3 ) of the contact discontinuity, ut2. That is,

$$
x_{R}\left(t_{2}\right)=\left(u+c_{F}^{\prime}\right)\left(t_{2}-t_{1}^{\prime}\right)+v_{o} t_{1}^{\prime}-d_{F}<u t_{2}
$$

\footnotetext{
${ }^{4}$ The cushion can be further he ated if the reflected shock is itself reflected at the contact discontinuity back into the cushion. If the shock is not reflected, a rarefaction will travel back into the cushion causing disassembly and conversion of the stored internal energy into kinetic energy. These cases are not considered in the analysis above.
} 
where $c_{F}$ is the sound speed in the driven after it is shocked, $t_{1}{ }^{\prime}$ is the time for the shock to reach the outside of the driver, and is given by

$$
t_{1}^{\prime}=\frac{d_{F}}{v_{o}+\left|U_{F}\right|}
$$

and

$$
t_{2}=\frac{d_{c}+\left|U_{C, 2}\right| t_{1}}{u+\left|U_{C, 2}\right|}
$$

In the special case that $\gamma_{C}=\gamma_{F}=\gamma$ and $\rho_{o, C}=\rho_{o, F}=\rho_{o}$, we find

$$
c_{F, 1}=\frac{\nu_{o}}{2} \sqrt{\frac{\gamma(\gamma-1)}{2}}
$$

and the inequality places the following condition on the dimensions of the initial assembly:

$$
\frac{d_{C}}{d_{F}}<\frac{\gamma}{3 \gamma-1}\left[2+\sqrt{\frac{2(\gamma-1)}{\gamma}}\right]
$$

\section{RADIATION-HYDRODYNAMIC ASSEMBLY OF THE FRC}

In the preceeding section we considered the hydrodynamic conditions in the driver and the cushion after assembly. Prior to assembly, ultraviolet radiation from the Joule-heated imploding driver will preheat the cushion. The shock waves produced when the driver impacts the cushion further heats the plasma (as discussed in Section III) and will serve as an additional source of radiation. The radiation front produced by the shock in the cushion can be described by similarity solutions to the radiation hydrodynamic equations prior to its reaching the axis of symmetry (the impenetrable wall in the planar model). Once radiation reaches the wall the equations must in general be solved numerically. We are primarily interested in the case where the radiation field moves supersonically into the cushion (material and radiation in the cushion ahead of the shock are not strongly coupled). For real materials the effective gammas, $\gamma_{C}$ and $\gamma_{F}$, and the specific heat 
appearing in (6) are not constants, but vary with ionization during the evolution. This makes analytic treatments difficult. Consequently, an analysis including radiative effects has been carried out using a one dimensional (1-D) hydrodynamic computational model which includes radiation diffusion and material coupling in a three temperature approximation and uses tabular equations of state and opacities for the driver and liner. 5 The effects of magnetic fields are not included in this discussion (this allows us to concentrate on the radiation and hydrodynamic issues). The presence of MHD effects will be considered in a subsequent paper. Thus we exclude the effects of Joule heating of the drive plasma during assembly and the magnetic tamping of the pinch. These effects are expected to increase the temperature of the driver and extend the time of maximum temperature in the cushion by reducing the amount of expansion at pinch. Preliminary 1-D MHD calculations using tabular electrical resistivities for carbon and hydrogen in the cushion indicate that there is little current penetration into the cushion during assembly and after pinch.

The accuracy of the numerical hydrodynamic treatment has been confirmed by benchmarking it against the analytic solutions presented in Section III. The test problem consisted of two gamma law fluids in planar geometry. The cushion was $0.5 \mathrm{~cm}$ thick, with an initial density $0.053 \mathrm{~g} / \mathrm{cm}^{3}$. We used (1) for the equation of state for the cushion, with $\gamma_{c}=1.4$. The cushion was initially at rest with essentially zero internal energy. The driver for the FRC was $0.5 \mathrm{~cm}$ thick, with an initial density $0.0265 \mathrm{~g} / \mathrm{cm}^{3}$. The equation of state for the FRC was of the form (1) with $\gamma_{F}=5 / 3$. The entire FRC had an initial velocity of $40 \mathrm{~cm} / \mu$ s and essentially zero internal energy. The calculated and theoretical values for the fluid variables associated with the main shock and the first reflected shock compared quite well, as is shown in Table 1.

The driver plasma for the numerical model is gold and the cushion is $\mathrm{CH}$ foam with initial conditions shown in Figure 5. The initial conditions chosen for the gold plasma are typical of the conditions obtained in MHD simulations of a thin gold foils driven by pulsed power systems. Unperturbed models tend to predict a relatively thin radial sheath near pinch. However, perturbed

${ }^{5}$ A description of the physics model used here can be found in D.L. Peterson, R.L. Bowers, J.H. Brownell, A.E. Greene, K.D. Mclenithan, T.A. Oliphant, N.F. Roderick and A.J. Scannapieco, to appear in Phys. Plasmas, Jan. 1996. 
(2-D) simulations produce a much thicker plasma near pinch. These effects will reduce the temperature in the cushion. We have chosen the thickness of the driver plasma to reflect these effects. Tabular constitutive relations have been used in the simulations. Figure 4 shows the equation of state $P(\rho, T)$ and the Rosseland mean opacity $\kappa(\rho, T)$ used for each of these materials. The initial temperatures of each material are also chosen to represent the conditions from MHD simulations at impact, which include the radiative losses and Joule heating of the driver plasma during implosion, and a simple (spatially uniform) pre-heat model for the cushion. Figure 6 shows the position versus time of the radiation front (solid curve), the contact discontinuity (dashed-dot curve), the shock front in the cushion (dotted curve) and the shock front in the gold (dashed curve).

At impact, shock waves are generated in the driver and in the cushion which can be described by the analysis of Section III. Shock heating of the plasma serves as the source of radiation generation in the hohlraum and of radiative losses from the driver plasma. In this section we consider the dynamics of the driver and the cushion, and concentrate on radiative effects. Profiles of density (in $\mathrm{g} / \mathrm{cm}^{3}$ ), radiation temperature (in $\mathrm{eV}$ ) and material temperature (in $\mathrm{eV}$ ) versus the spatial coordinate $x($ in $\mathrm{cm}$ ) are shown in Figure 7 at several times after impact $(\mathrm{t}=0)$. In the following sections we shall discuss the radiation-hydrodynamic effects using the temporal stages appearing in Section III.

\section{A. Assembly of the Hohlraum $\left(0<t<t_{1}\right)$}

At impact, the inward moving shock sets up a radiation front in the cushion which propagates toward the left with a velocity of about $-21 \mathrm{~cm} / \mu \mathrm{s}$. Figure 7 a shows system $5.3 \mathrm{~ns}$ after impact. The cushion density behind the shock is about $0.19 \mathrm{~g} / \mathrm{cm}^{3}$. The contact discontinuity is at $x_{c}=$ $0.41 \mathrm{~cm}$, and travels to the left with velocity $\mathrm{v}_{\mathrm{c}}=-16.4 \mathrm{~cm} / \mu \mathrm{s}$; the shock front, located at $\mathrm{x}_{\mathrm{c}, \mathrm{s}}=$ $0.38 \mathrm{~cm}$, travels with velocity $\mathrm{v}_{\mathrm{S}}=-20 \mathrm{~cm} / \mu \mathrm{s}$. The Rosseland mean free path in the unshocked portion of the cushion initially is about $2.5 \times 10^{-3} \mathrm{~cm}$. As the radiation front moves into it, the cushion is preheated to about $50 \mathrm{eV}$ and the mean free path increases to about $0.04 \mathrm{~cm}$. Here, the radiation field and the cushion are only weakly coupled. At the front the radiation temperature $T_{R}$ 
$=90 \mathrm{eV}$, and the plasma temperature $T_{e}=55 \mathrm{eV}$. Behind the shock front in the cushion $T_{R}$ varies from $125 \mathrm{eV}$ to $135 \mathrm{eV}$, and $\mathrm{T}_{\mathrm{e}}=140 \mathrm{eV}$ (apart from the small peak at the shock front).

In the FRC the radiation and plasma are tightly coupled. The radiation diffusion front moves supersonically outward from the contact discontinuity at about $17.3 \mathrm{~cm} / \mu \mathrm{s}$, reaching the backside of the driver at $t=10 \mathrm{~ns}$. This corresponds to the beginning of the $\mathrm{x}$-ray emission from the FRC as seen to the right. In a cylindrical pinch this would correspond to the radial output. The peak power during this stage occurs at about $\mathrm{t}=16.9 \mathrm{~ns}$ (Figure 8), and is $55.9 \mathrm{MJ} / \mu \mathrm{s}$. The total output per unit area ${ }^{6}$ during this stage of the implosion is about $175 \mathrm{~kJ} / \mathrm{cm}^{2}$.

Near the end of first stage of $x$-ray output, the FRC appears as shown in Figure $7 \mathrm{~b}$. The radiation temperature in the cushion varys from about $75 \mathrm{eV}$ to $125 \mathrm{eV}$, and preheats the cushion to about $50 \mathrm{eV}$. The shock-heated cushion is at a plasma temperature of about $130 \mathrm{eV}$. Just prior to the time the shock reaches the wall $(x=0)$ the cushion has been heated to a nearly uniform temperature of about $125 \mathrm{eV}$. During this period the driver is about 500 radiation mean free paths thick, and acts as an efficient radiation case. The opacity in the shock heated cushion is about 600 $\mathrm{cm}^{2} / \mathrm{g}$, corresponding to a Rosseland mean free path of about $10^{-2} \mathrm{~cm}$. This corresponds to only a few radiation mean free paths between the wall and the contact discontinuity. ${ }^{7}$ This explains the near uniform radiation field structure in the shocked cushion. The relative lack of $\mathrm{x}$-ray output on the right from the right (Figure 8) during the time $20 \mathrm{~ns}<\mathrm{t}<32 \mathrm{~ns}$ shows that the FRC is acting to confine the radiation.

\section{B. Shock Reflection $\left(t_{1}<t<t_{2}\right)$}

The shock in the cushion is reflected from the wall at about $24 \mathrm{~ns}$. Figure $7 \mathrm{c}$ shows the structure at $\mathrm{t}=24.4 \mathrm{~ns}$ as the reflected shock begins to move to the right into the cushion. There is a rapid rise in plasma temperature at the wall $x=0$, which is weakly coupled to the radiation field. As during the first shock stage, the radiation from reflected shock moves ahead of the shock and preheats the plasma. This is clear in Figure $7 \mathrm{~d}$, showing the system at $\mathrm{t}=27.4 \mathrm{~ns}$ when the

\footnotetext{
6In the one-dimensional planar approximation the output is characterized by the emission per unit area of the system, and will be expressed in $\mathrm{MJ} / \mathrm{cm}^{2}$.

${ }^{7}$ The Rosseland mean opacity actually underestimates the mean free path in regions that are optically thin. Multigroup opacities would predict less absorption that indicated by the model here.
} 
reflected shock has reached $0.05 \mathrm{~cm}$. The temperature of the radiation precursor is slightly greater than $200 \mathrm{eV}$, and has heated the cushion to about $175 \mathrm{eV}$. The contact discontinuity is at $\mathrm{x}=0.1$ cm.

\section{Stagnation $\left(t_{2}<t\right)$}

Reflected shock heating of the cushion is complete at about $\mathrm{t}=31 \mathrm{~ns}$, and the second stage of $\mathrm{x}$-ray output begins. The structure of the FRC at $\mathrm{t}=31.32$ (Figure 7e) shows the contact discontinuity at about $x=0.075 \mathrm{~cm}$. The temperature in the cushion is slightly greater than $200 \mathrm{eV}$ (the radiation temperature is uniform). The second stage of $x$-ray output (Figure 8), which occurs as the reflected shock heats the driver, lasts about $5 \mathrm{~ns}$ and emits an additional $76 \mathrm{~kJ} / \mathrm{cm}^{2}$. The peak power during this stage, which occurs at about $t=34 \mathrm{~ns}$ ) is comparable to the first stage (about $55 \mathrm{MJ} / \mu \mathrm{s}$ ). The total $\mathrm{x}$-ray energy emitted to the right is about $251 \mathrm{~kJ} / \mathrm{cm}^{2}$. Including the MHD drive is expected to increase the energy output because the magnetic field acts effectively as a tamper for the system. This reduces cooling associated with the hydrodynamic expansion of the the FRC. Joule heating of the driver will also contribute to the net $\mathrm{x}$-ray output.

The energy partition during the implosion versus time is shown in Figure 9. The total energy in the radiation field is negligable compared with the other forms of energy and is not shown. The values shown assume a $1 \mathrm{~cm}$ radius disk for the driver and cushion. This corresponds to a $3.3 \mathrm{MJ}$ implosion, and produces about $0.8 \mathrm{MJ}$ of $\mathrm{x}$-ray output to the right.

Figure 10 shows the time history of the radiation temperature and the plasma temperature in the cushion at several points to the right of the wall. The point nearest the wall (solid curve) shows a slight precursor followed by a rapid rise to a peak temperature of about $250 \mathrm{eV}$. The other curves, which lie to the right of the wall, show the effect ot the first shock, which heats the plasma to about $140 \mathrm{eV}$, followed by the reflected shock which heats it to about $200 \mathrm{eV}$. Finally, Figure 11 shows the plasma pressure just to the right of the wall versus time.

\section{DISCUSSION}


The analytic hydrodynamic and numerical radiation-hydrodynamic models discussed above illustrate most of the essential physics of a FRC. More nearly realistic models should include the effects of magnetic fields and the magnetic drive during the implosion, as well as the effects of convergence associated with cylindrical geometry. Finally, the effects of magnetic RayleighTaylor instabilities during the implosion need to be included. Probably the most important of these issues are the effects of instability growth, since they tend to increase the thickness of the driver plasma and distribute the implosion kinetic energy over a larger volume. This leads to an increase in the radiation pulse-width at pinch. If the instabilities are of sufficient magnitude, they may disrupt the driver and seriously degrade the performance of the FRC. When one-dimensional calculations of bare implosions (a driver plasma without a central target) have been carried out and compared with experiments which are known to be perturbed, the agreement with data is not good (calculated radiation pulse-widths, for example, are too short and predicted plasma temperatures are too high). Two-dimensional studies of bare implosions which include the effects of instability growth have been carried out in the low energy regime ${ }^{8}$ and are found to resolve many of the discrepancies with the data. Similar two-dimensional studies of the FRC are needed to determine the effectiveness of this concept.

\section{APPENDIX}

The Hugoniot relations may be obtained in the laboratory frame of reference starting with the hydrodynamic equations of continuity, momentum and energy. In one spatial dimension the latter are

$$
\frac{\partial \rho}{\partial t}+\frac{\partial}{\partial x}(\rho u)=0
$$

\footnotetext{
${ }^{8}$ D.L. Peterson, R.L. Bowers, J.H. Brownell, A.E. Greene, K.D. McLenithan, T.A. Oliphant, N.F. Roderick, and A.J. Scannapieco, "Two-Dimensional Modeling of Magnetically Driven Rayleigh-Taylor Instabilities in Cylindrical Z Pinches", Physics of Plaasmas 3, 368 (1996); W. Matuska, R.L. Bowers, J.H. Brownell, H. Lee, C.M. Lund, D.L. Peterson and N.F. Roderick, "Two-Dimensional Modeling of XRadiation Output from Perturbed Z Pinches", Physics of Plasmas 3, 1415 (1996).
} 


$$
\begin{gathered}
\frac{\partial u}{\partial t}+u \frac{\partial u}{\partial x}=-\frac{l}{\rho} \frac{\partial P}{\partial x} \\
\frac{\partial}{\partial t}\left(\rho \Sigma+\frac{\rho u^{2}}{2}\right)=-\frac{\partial}{\partial x}\left[\rho u\left(\Sigma+\frac{u^{2}}{2}\right)+P u+S\right]
\end{gathered}
$$

where $\rho, u$, and $P$ are the material density, velocity and pressure, respectively. The quantity $\Sigma$ is the specific internal energy, and $S$ is the total radiative flux. We assume that the shock structure is steady and transform to a frame that is moving at the shock speed, $U>0$. In this coordinate system the flow variables are independent of time, and under the transformation $x^{\prime}=x-U t, t^{\prime}=t \quad$, equations (1)-(3) become

$$
\frac{d}{d x}[(u-U) \rho]=0, \quad \frac{d}{d x}[\rho u(u-U)+P]=0
$$

and

$$
\frac{d}{d x}\left[\rho\left(\Sigma+\frac{u^{2}}{2}\right)(u-U)+P u+S\right]=0
$$

It will be assumed that the shock front and the radiation front extend only over an inifinitesimal width (a few mean-free paths). In fact, it is found that the front has a width of several photon mean-free paths. Integrating these equations, and evaluating the integration constants far away from the shock on the downstream side where $\mathrm{u}=0, P=P_{o}, \Sigma=\Sigma_{o}$ and $S=S_{o}$, we obtain:

$$
\begin{gathered}
(U-u) \rho=U \rho_{o} \\
\rho_{o} U u=P-P_{o} \\
\rho_{o} U\left(\Sigma-\Sigma_{o}+\frac{u^{2}}{2}\right)=P u+S-S_{o}
\end{gathered}
$$


where $\rho, u, P$, and $\Sigma$ denote quantities behind the shock and the subscript o denotes quantities in front of the shock. Equations (4)-(6) are the Hugoniot relations in the laboratory frame of . reference, which have been used to derive the results in Section III.

Finally, we consider the flow variables away from the shock front when radiation is present. In particular, we show that the flow variables associated with the shock, which is strong enough that radiative flux must be accounted for, approaches those in a shock where radiation can be ignored. Denoting flow variables in the former case (radiation present) by primes, and using unprimed quantities for the corresponding variables without radiation, we write the energy relation along the Hugoniot (6) as

$$
\begin{aligned}
& \rho_{o} U^{\prime}\left(\Sigma^{\prime}-\Sigma_{o}^{\prime}+\frac{u^{\prime 2}}{2}\right)=P^{\prime} u^{\prime}+S(x) \\
& \rho_{o} U\left(\Sigma-\Sigma_{o}+\frac{u^{2}}{2}\right)=P u
\end{aligned}
$$

Subtracting the second from the first equation gives

$$
\rho_{o} U^{\prime}\left(\Sigma^{\prime}-\Sigma_{o}^{\prime}\right)-\rho_{o} U\left(\Sigma-\Sigma_{o}\right)+\rho_{o} U^{\prime} \frac{u^{\prime 2}}{2}-\rho_{o} U \frac{u^{2}}{2}=P^{\prime} u^{\prime}-P u+S(x)
$$

Next, we note that the specific internal energy $\Sigma_{o}{ }^{\prime}=\Sigma_{o}$, so the second term on the left side vanishes. Now we assume that away from the front, the radiation field is in equilibrium and the positive and negative components of the flux cancel. Therefore $S \rightarrow 0$ at points removed from the discontinuity. Of course, this does not necessarily imply that the actual values of the fluid variables have not been altered from their non-equilibrium values. We can also subtract the mass continuity equation for non-radiative flow from the corresponding equation including radiation to obtain

$$
P_{d}\left(U^{\prime}-U\right)=\left(U^{\prime}-u^{\prime}\right) \rho^{\prime}-(U-u) \rho
$$


In the same way we find for the momentum equation

$$
\rho_{o}\left(U^{\prime} u^{\prime}-U u\right)=P^{\prime}-P
$$

At large distances (compared with the mean free paths) behind the shock front we have $u=u^{\prime}$ and $P=P^{\prime}$, since these quantities are set by the piston (ignoring the short time interval when the front is near the piston when the radiative flux can affect the fluid variables). It then follows from the fluid equation of state (and the assumption that the fluid is in radiative equilibrium) that $\Sigma=\Sigma$ ' away from the front. Consequently, away from the front the flow variables approach their radiation-free values. These assumptions have been used throughout the analysis of Section III. 
Table 1. Comparison of calculated and theoretical values of fluid variables in hydrodynamic test problem. The subscripts 0,1 , and 2 denote initial quantities, quantities behind the first shock, and quantities behind the reflected shock respectively. Numbers in parenthesis are from the analytic model.

\begin{tabular}{llllllll}
\hline \multicolumn{2}{c}{$\rho 1 / \rho 0$} & $\mathrm{U}(\mathrm{cm} / \mu \mathrm{s})$ & $\mathrm{u}(\mathrm{cm} / \mu \mathrm{s})$ & $\rho 2 / \rho_{1}$ & $\varepsilon_{2} / \varepsilon_{1}$ & $\mathrm{U}_{\mathrm{c} 2}(\mathrm{~cm} / \mu \mathrm{s})$ \\
$\mathrm{Au}$ & $\mathrm{CH}$ & $\mathrm{Au}$ & $\mathrm{CH}$ & & & & \\
\hline 4.19 & 6.11 & 9.94 & 20.3 & 17.3 & 3.55 & 2.29 & 6.6 \\
$(4.0)$ & $(6.0)$ & $(9.47)$ & $(20.5)$ & $(17.1)$ & $(3.5)$ & $(2.29)$ & $(6.84)$ \\
\hline
\end{tabular}




\section{Figure Captions}

Figure 1. Schematic representation of a flying radiation case (FRC) in cylindrical geometry. In each case the voltage drop associated with the pulsed power source (not shown) is connected across the two electrodes. (a) FRC and a central cushion; (b) cushion containing a capsule; (c) cushion and a side hohlraum. The axis of symmetry is horizontal.

Figure 2. Schematic set up for planar model of FRC. A rigid wall is located at the left $\left(x=d_{C} \quad\right)$, and the contact discontinuity between the FRC and the cushion is located initially at $x=0$. The outer boundary of the FRC is denoted by $-d_{F}$. Initial values for the fluid variables are also shown. Finally, the locations $x_{C, s}$ and $x_{F, s}$ of the shock fronts are shown in the cushion and plasma respectively.

Figure 3. Space-time representation of the evolution of the FRC. The spatial coordinate $x$ runs vertically. The reflecting boundary is shown cross-hashed. The times $t_{1}^{\prime}, t_{1}$ and $t_{2}$ correspond to shock breakout at the outer surface of the driver, shock arrival at the reflecting wall, and the time the reflected shock in the cushion reaches the contact discontinuity.

Figure 4. Equations of state and opacities used in the radiation-hydrodynamic model of the

planar FRC. (a) $P(\rho, T)$ in Mbar and (b) Rosseland mean opacity $k(\rho, T)$ in $\mathrm{cm}^{2} \mathrm{~g}^{-1}$ for a gold FRC. Pressure isotherms range from $2.5 \times 10^{-3} \mathrm{eV}$ to $300 \mathrm{eV}$. The opacity curves range from $2 \times 10^{-5} \mathrm{~g} / \mathrm{cm}^{3}$ to $2 \times 10^{3} \mathrm{~g} / \mathrm{cm}^{3}$. (c) $P(\rho, T)$ and (d) Rosseland mean opacity $\kappa(\rho, \mathrm{T})$ for a $\mathrm{CH}$ cushion. The pressure isotherms range from $2.5 \times 10^{-3} \mathrm{eV}$ to $500 \mathrm{eV}$, and the opacity curves from $1 \times 10^{-6} \mathrm{~g} / \mathrm{cm}^{3}$ to $10^{4} \mathrm{~g} / \mathrm{cm}^{3}$. 
Figure 5. Initial conditions for the 1-D planar model of the FRC, showing the driver plasma and the cushion at the moment of impact. The driver is moving to the left with a kinetic energy of 1.04 $\mathrm{MJ} / \mathrm{cm}^{2}$. The left-hand boundary is considered to be impenetrable to matter and radiation.

Figure 6. Position versus time for the radiation hydrodynamic calculation of the radiation front in the cushion (solid curve), the contact discontinuity (dashed-dot curve), the shock in the cushion (dotted curve) and the shock in the gold driver (dashed curve).

Figure 7. Profiles of plasma density (solid curve, in $\mathrm{g} / \mathrm{cm}^{3}$ ), radiation temperature $T_{R}$ (dotted curve, in $\mathrm{eV}$ ) and material temperature $\mathrm{T}$ (dash-dot curve, in $\mathrm{eV}$ ) versus spatial coordinate $\mathrm{x}(\mathrm{cm}$ ) at several times: (a) $t=5.3 \mathrm{~ns}$; (b) $t=19.6 \mathrm{~ns}$; (c) $t=24.4 \mathrm{~ns}$; (d) $t=27.4 \mathrm{~ns}$; and (e) $t=31.3 \mathrm{~ns}$. Impact occurs at $t=0$, and the rigid boundary is located at $x=0$. The position of the contact discontinuity between the gold and the cushion for the times above is $x_{C D}=0.41 \mathrm{~cm}, 0.19 \mathrm{~cm}$, $0.14 \mathrm{~cm}, 0.09 \mathrm{~cm}$ and $0.08 \mathrm{~cm}$, respectively.

Figure 8. Radiation output (solid curve, in $\mathrm{MJ} / \mathrm{cm}^{2}$ ) and power (dotted curve, in $\mathrm{MJ} / \mu \mathrm{s}$ ) from the outside of the gold plasma, versus time in $\mu$ s. The power is normalized to the peak value of 58.87 $\mathrm{MJ} / \mu \mathrm{s}$, which occurs at $\mathrm{t}=16.9 \mathrm{~ns}$.

Figure 9. Energy (MJ) versus time ( $\mu \mathrm{s})$. Kinetic energy (solid), plasma internal energy (dotted), and radiation output $\mathrm{E}_{\text {out }}$ (dashed-dot). The dashed curve at top is the total energy in the problem, which should be a constant.

Figure 10. Material temperature in $\mathrm{keV}$ versus time in $\mu \mathrm{s}$ for several positions in the cushion near the rigid boundary.

Figure 11. Material pressure (Mbar) near the wall in the cushion versus time ( $\mu s)$. 


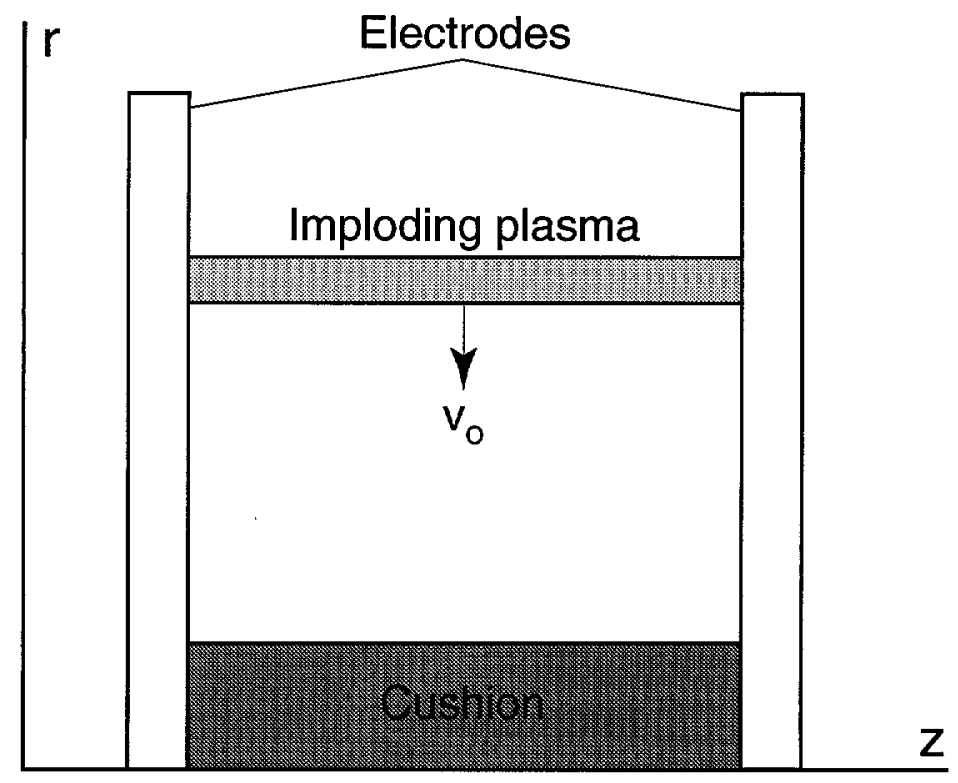

Figure 1a 


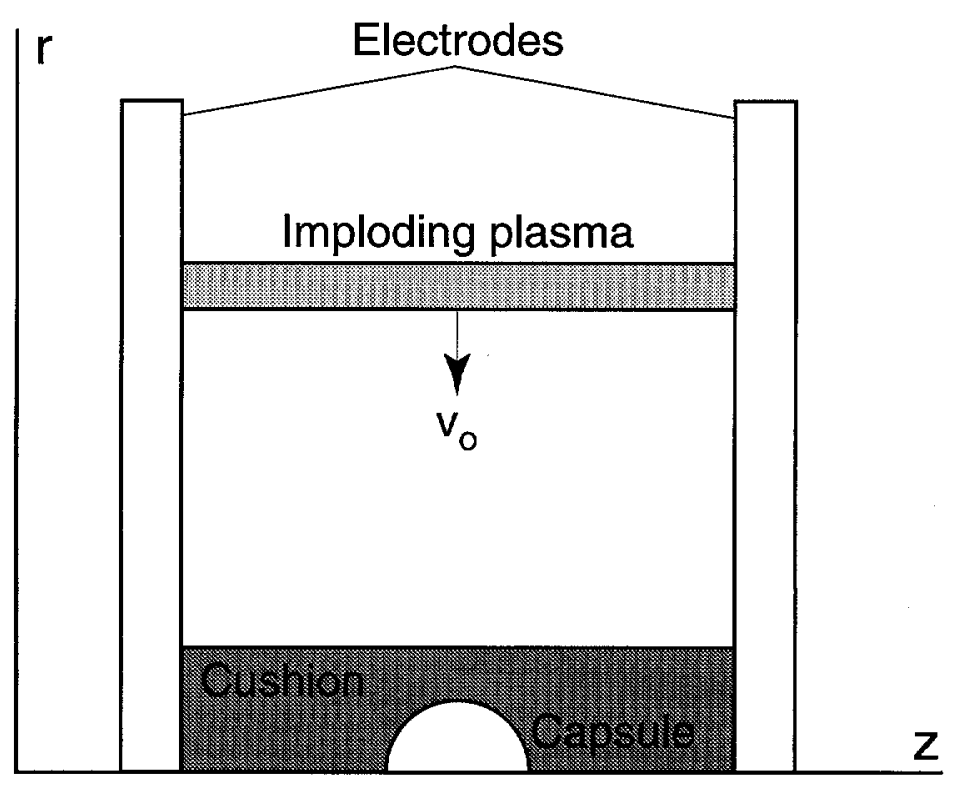

Figure $1 b$ 


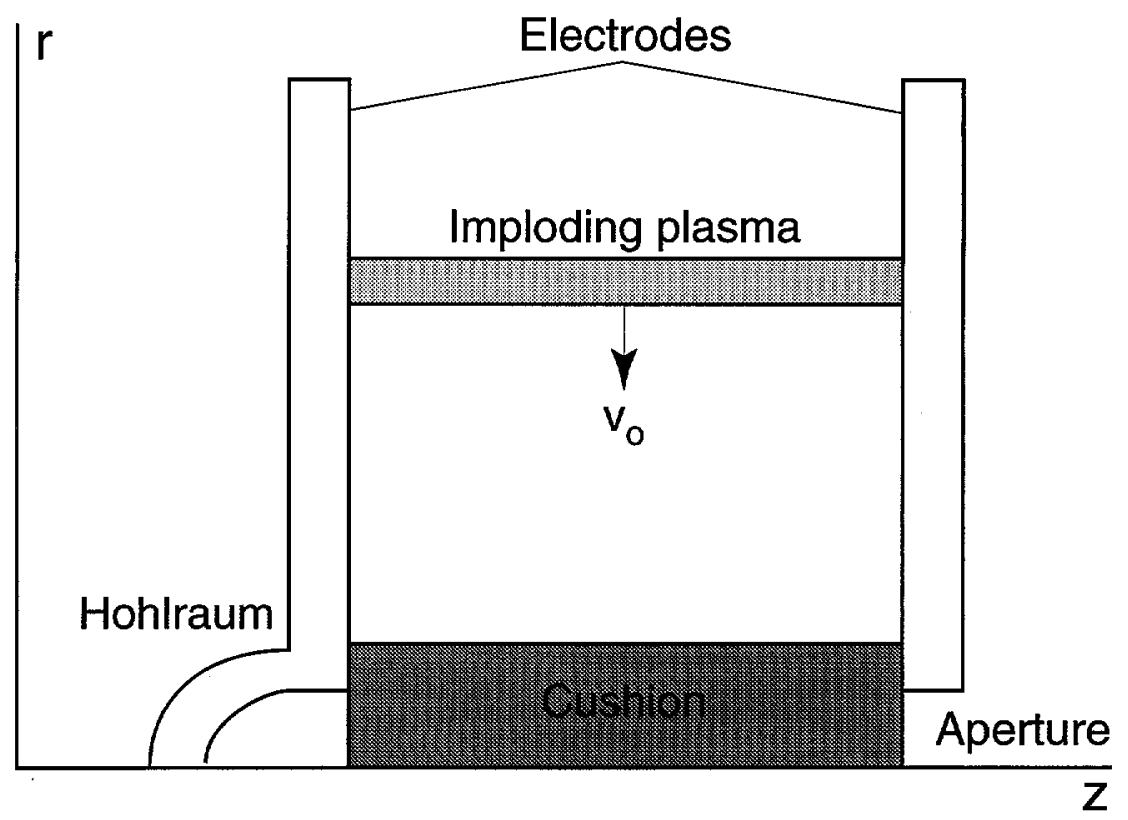

Figure 1c 


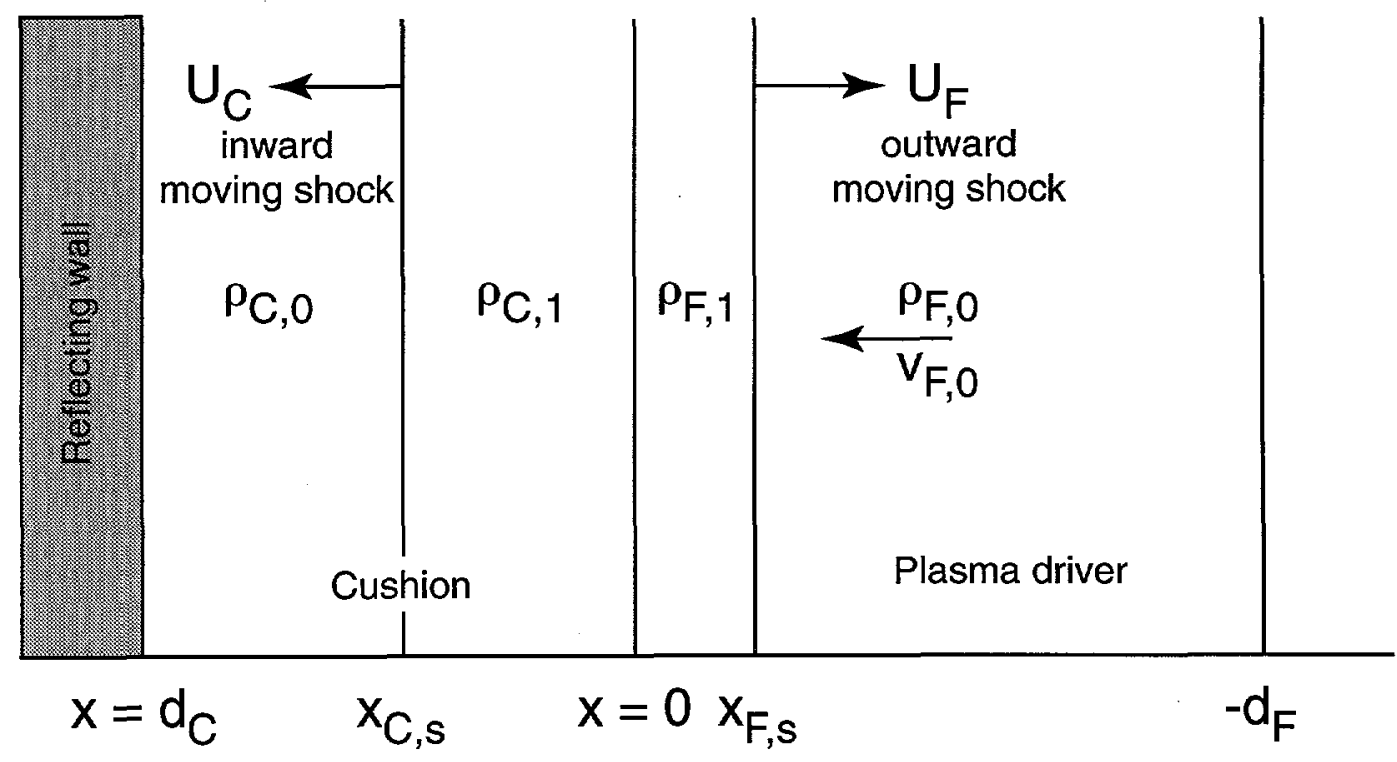

Figure 2 


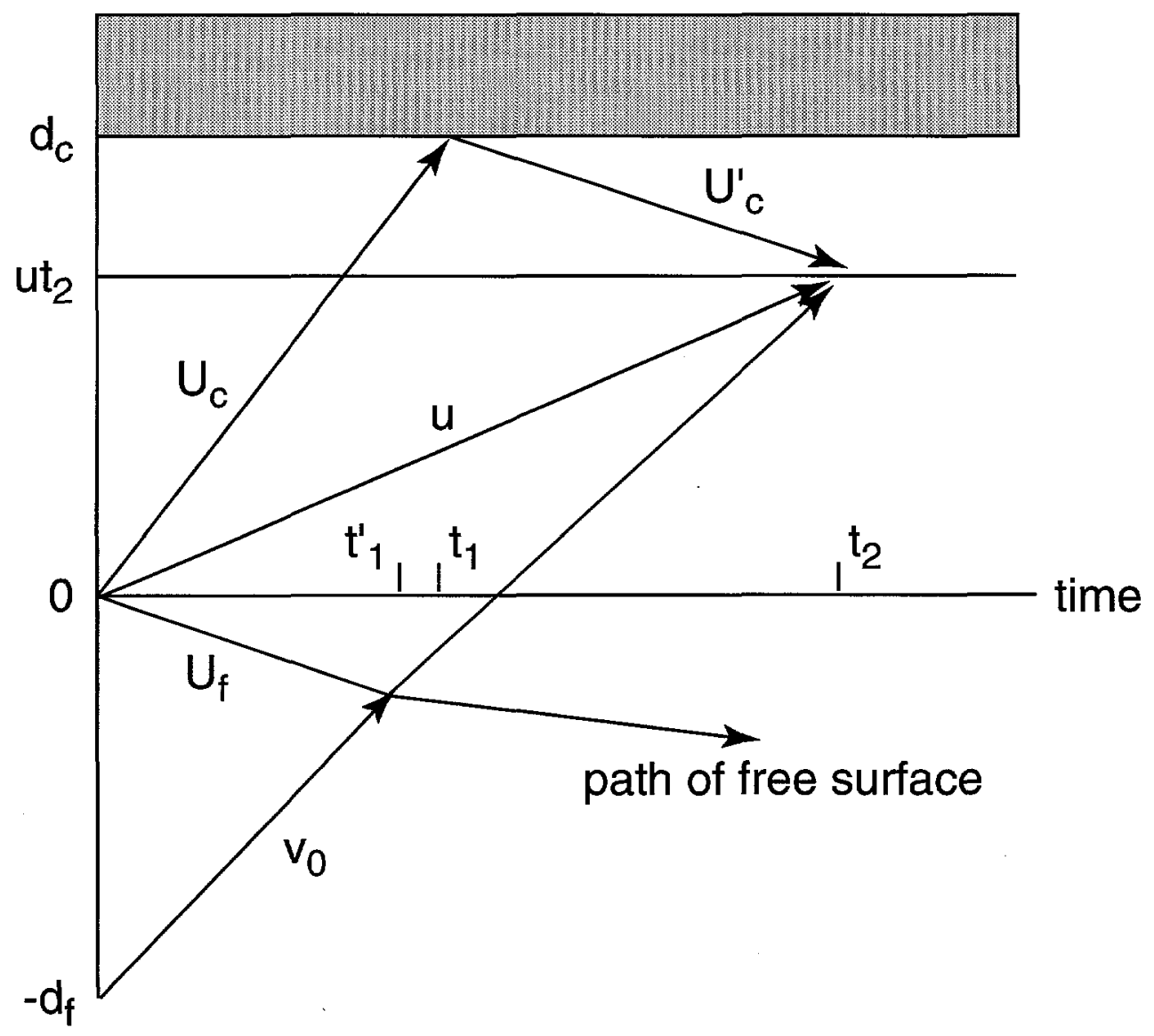

Figure 3 


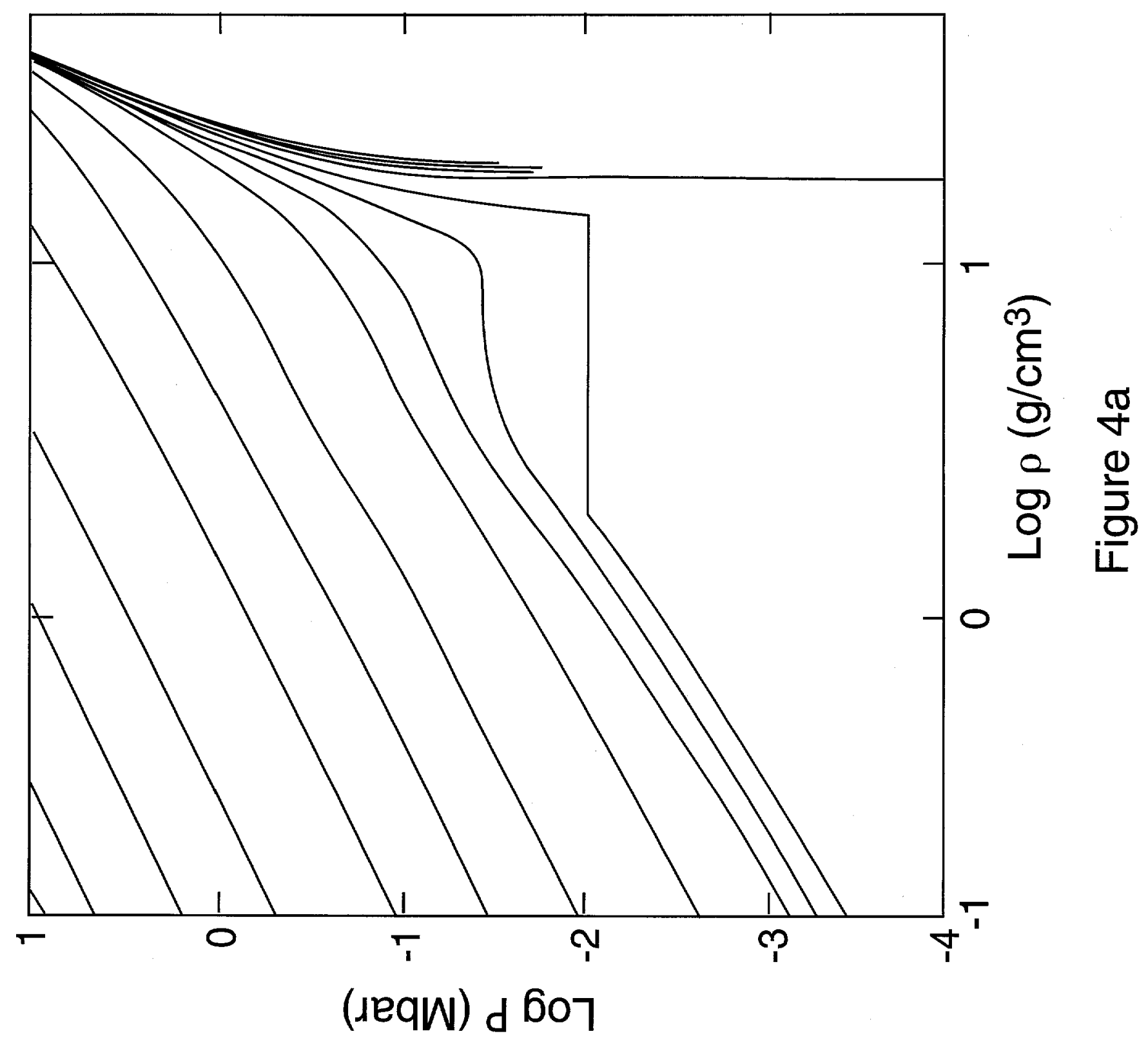




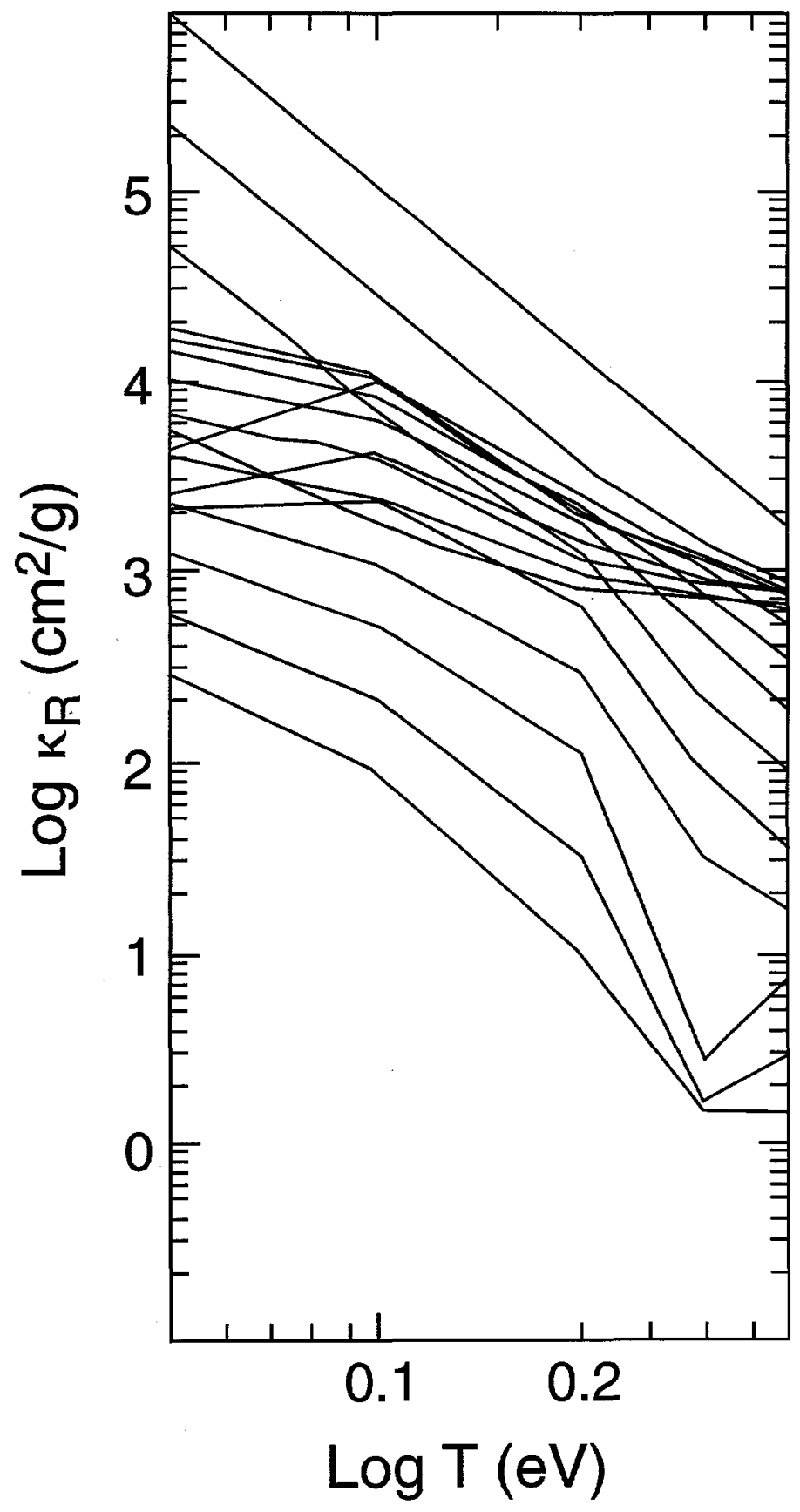

Figure 4b 


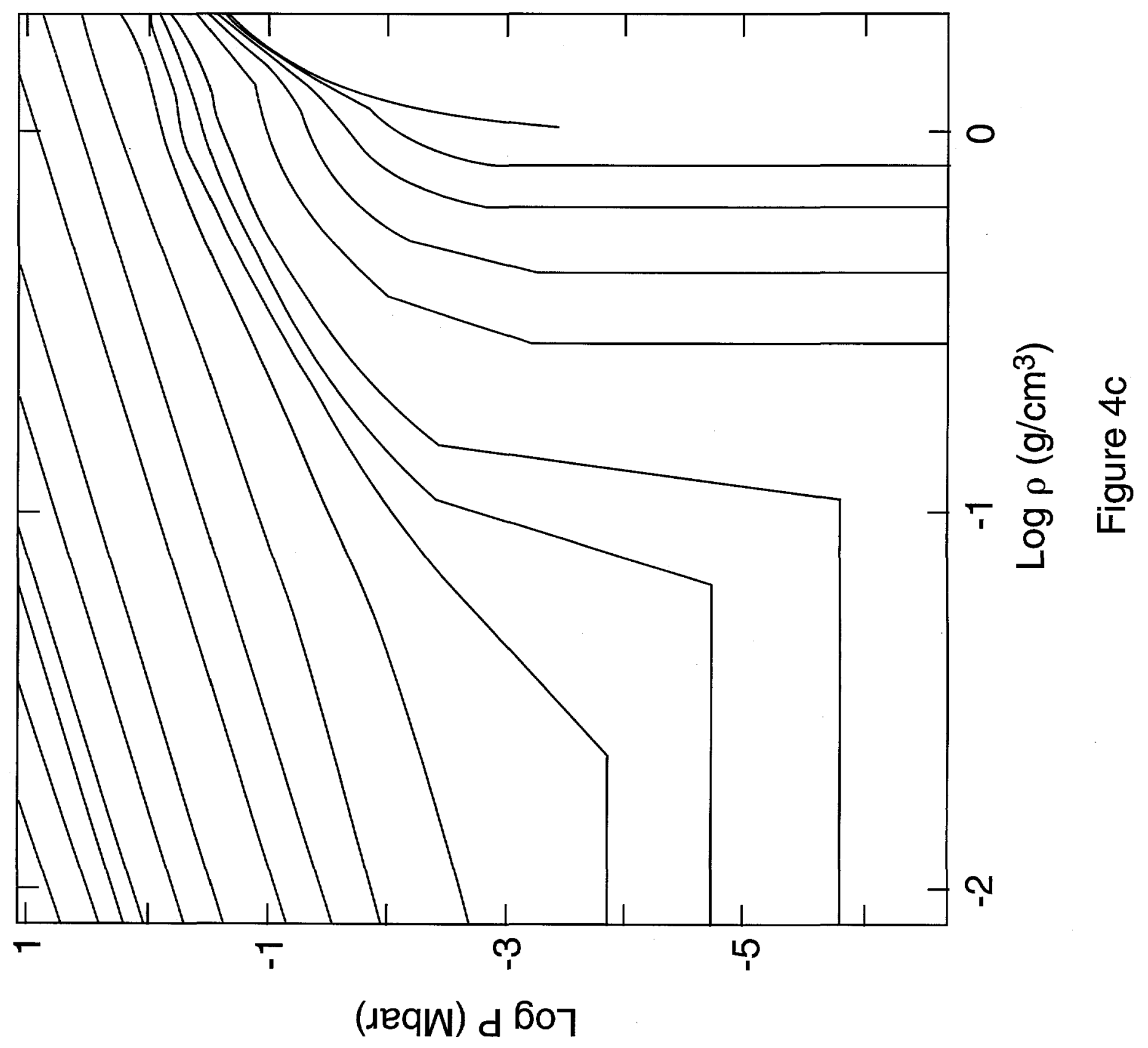

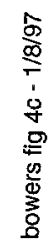




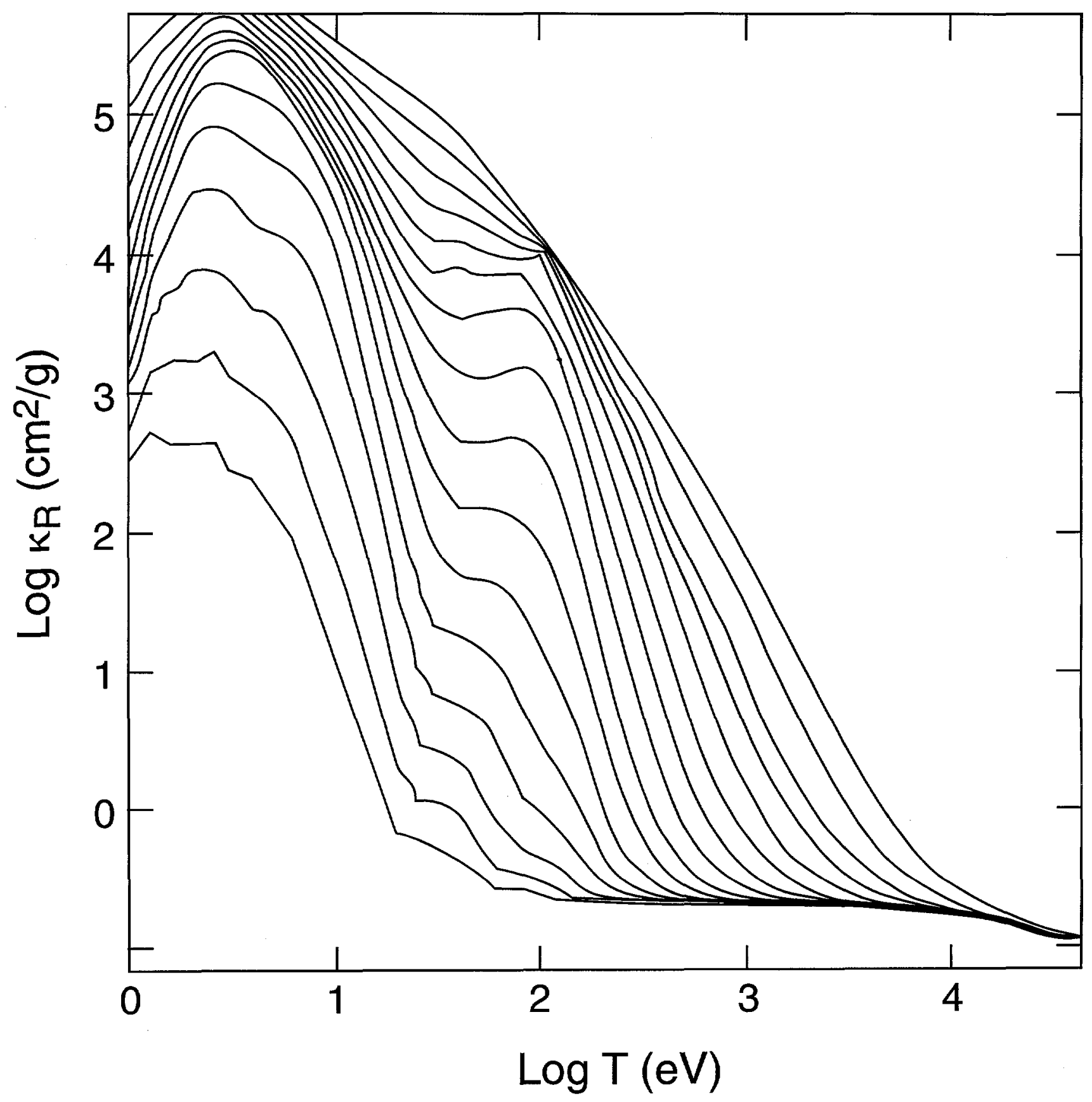

Figure 4d 


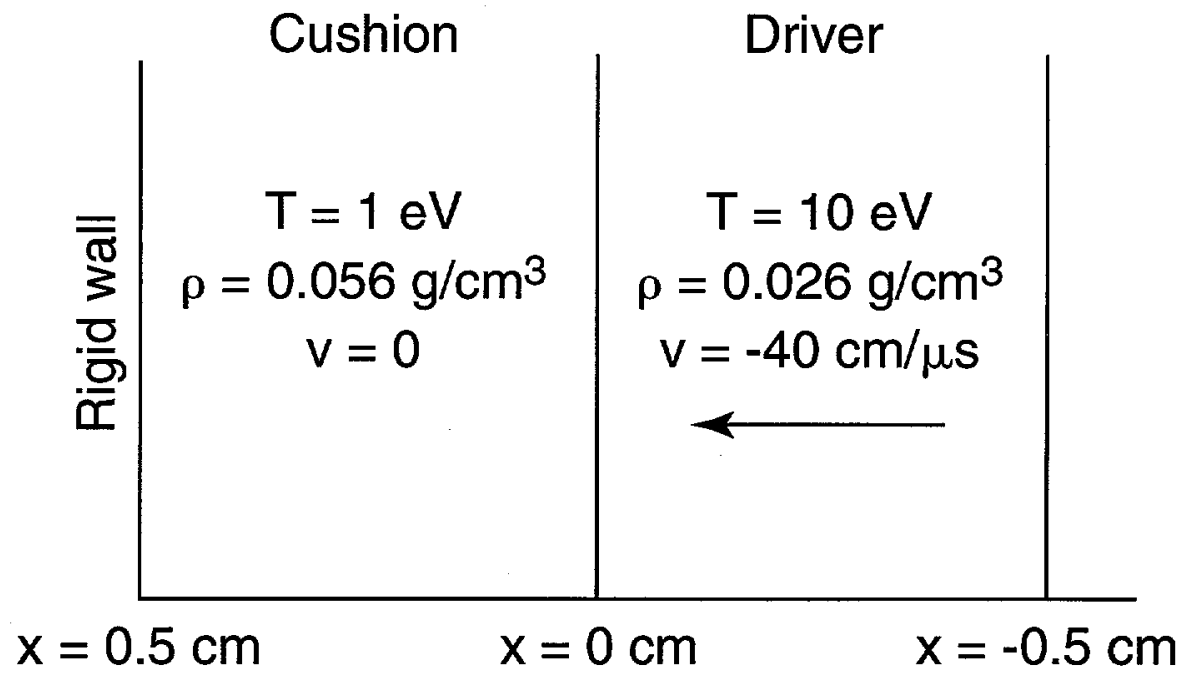

Figure 5 


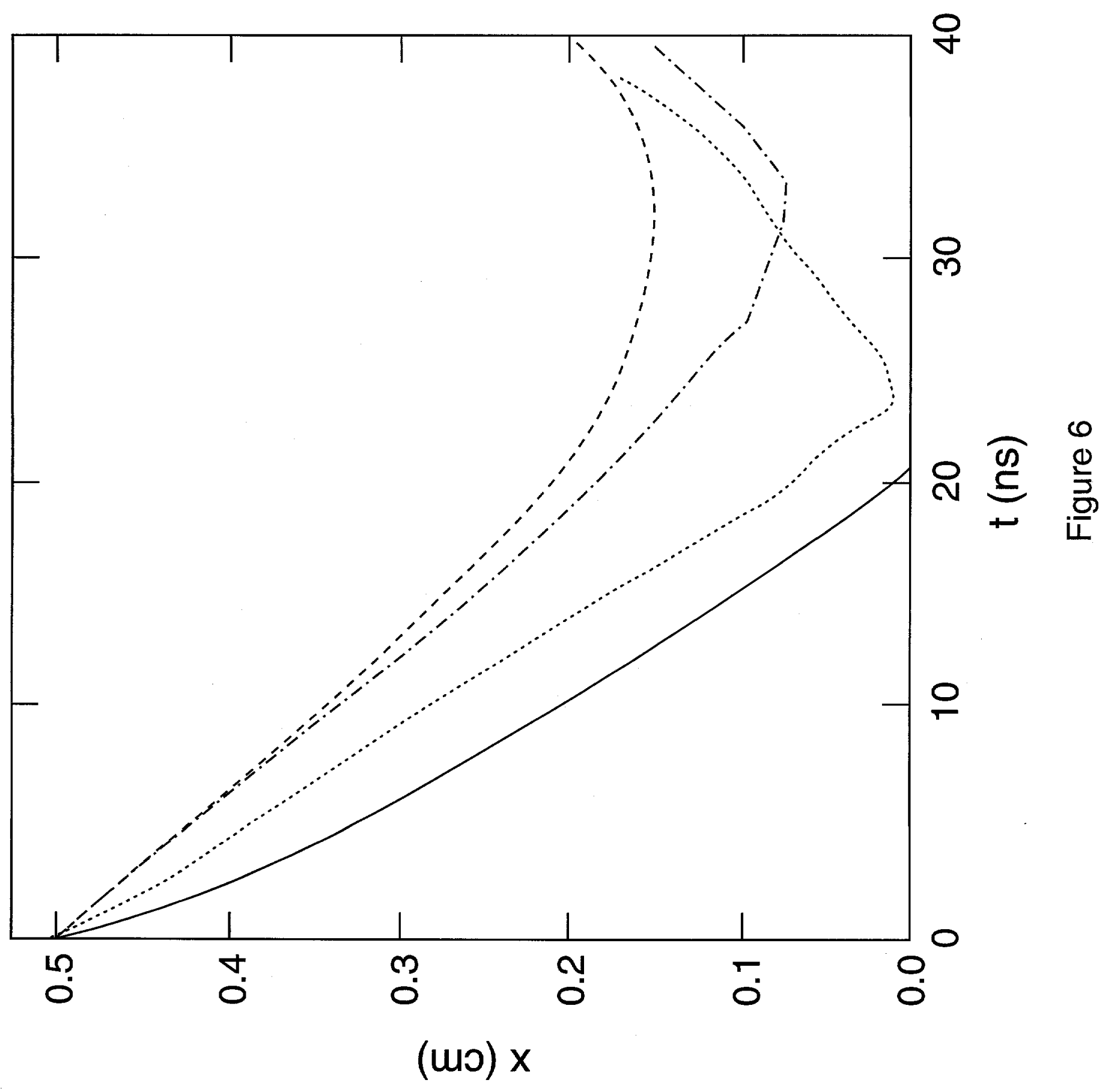




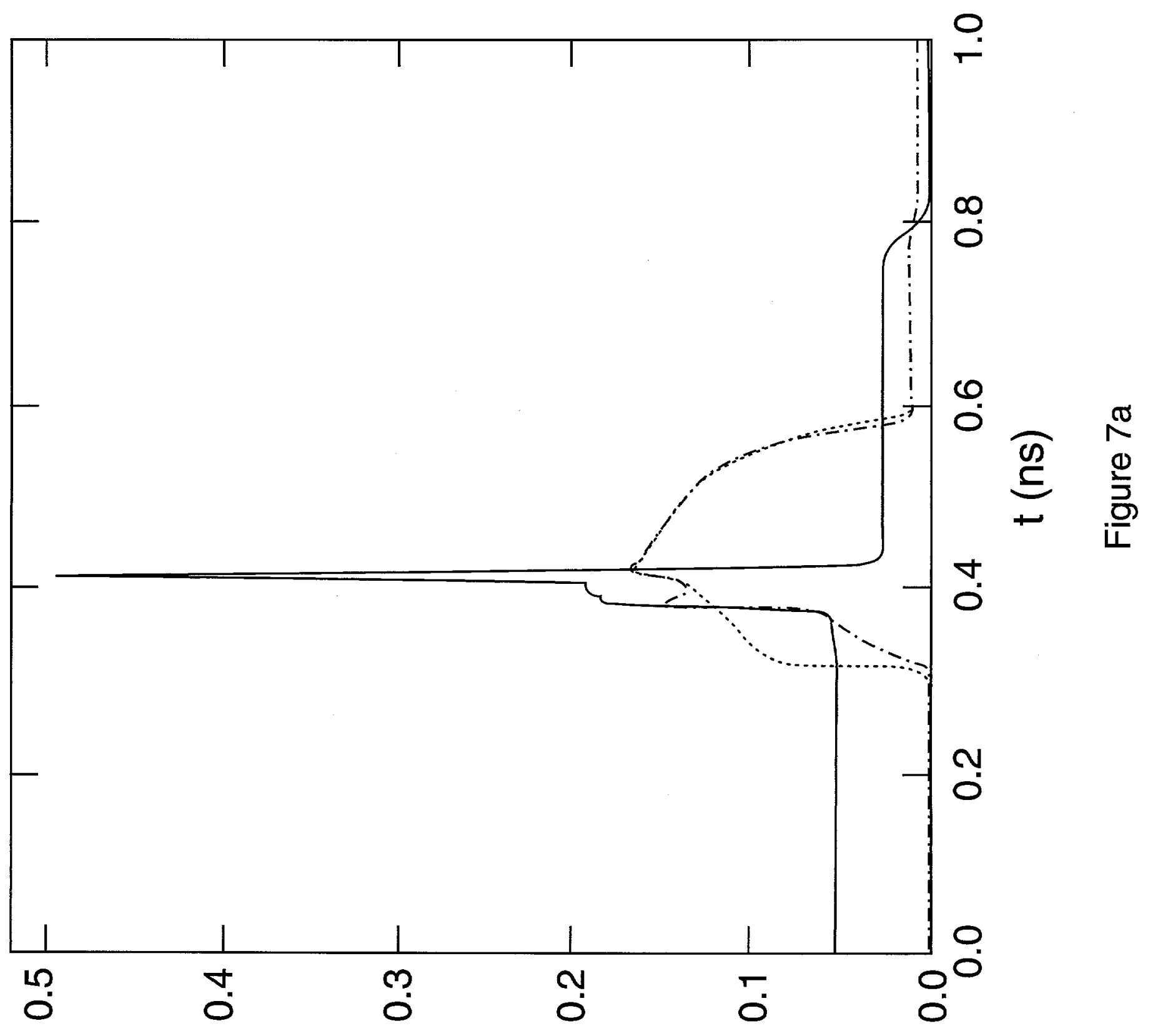




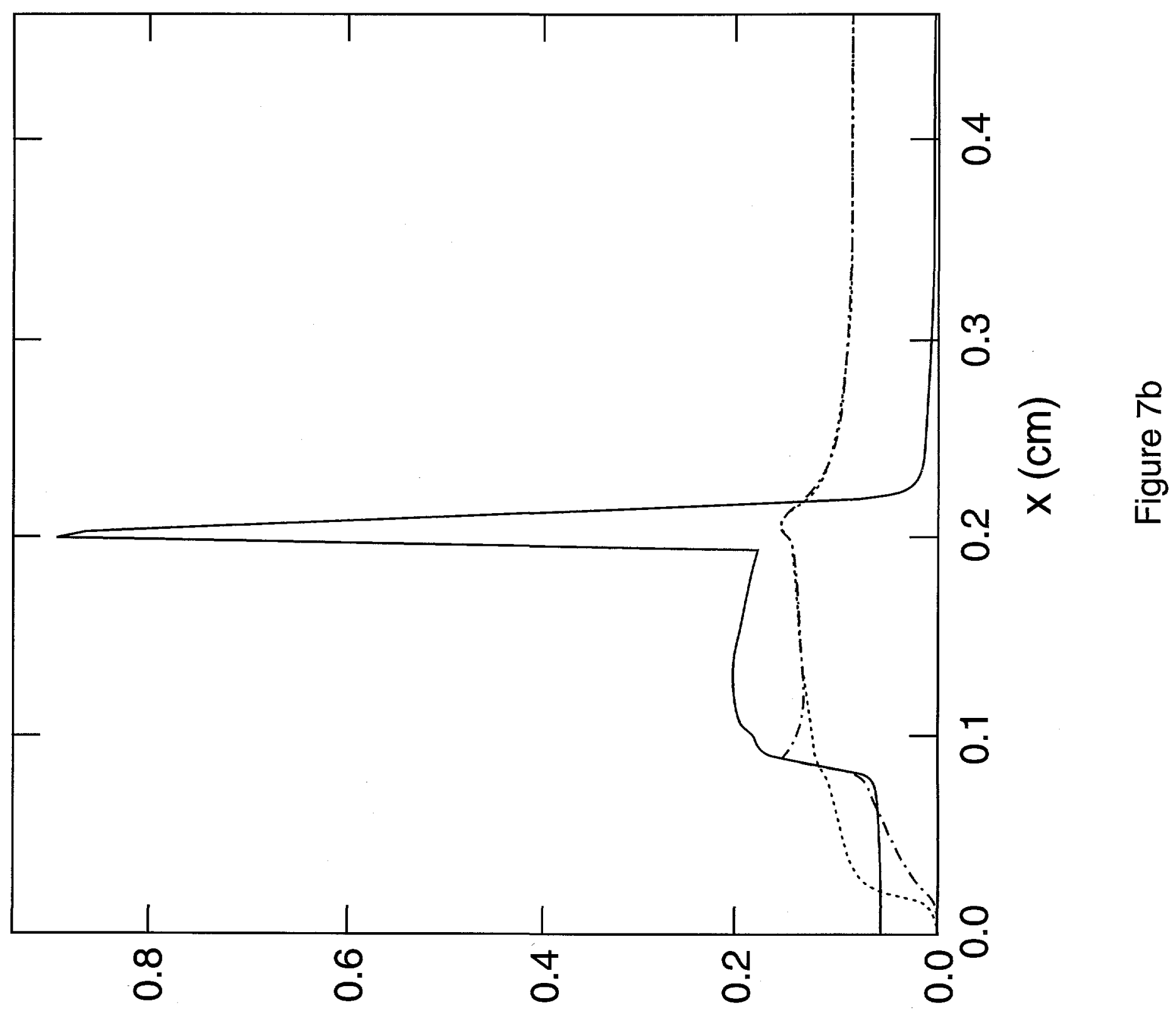




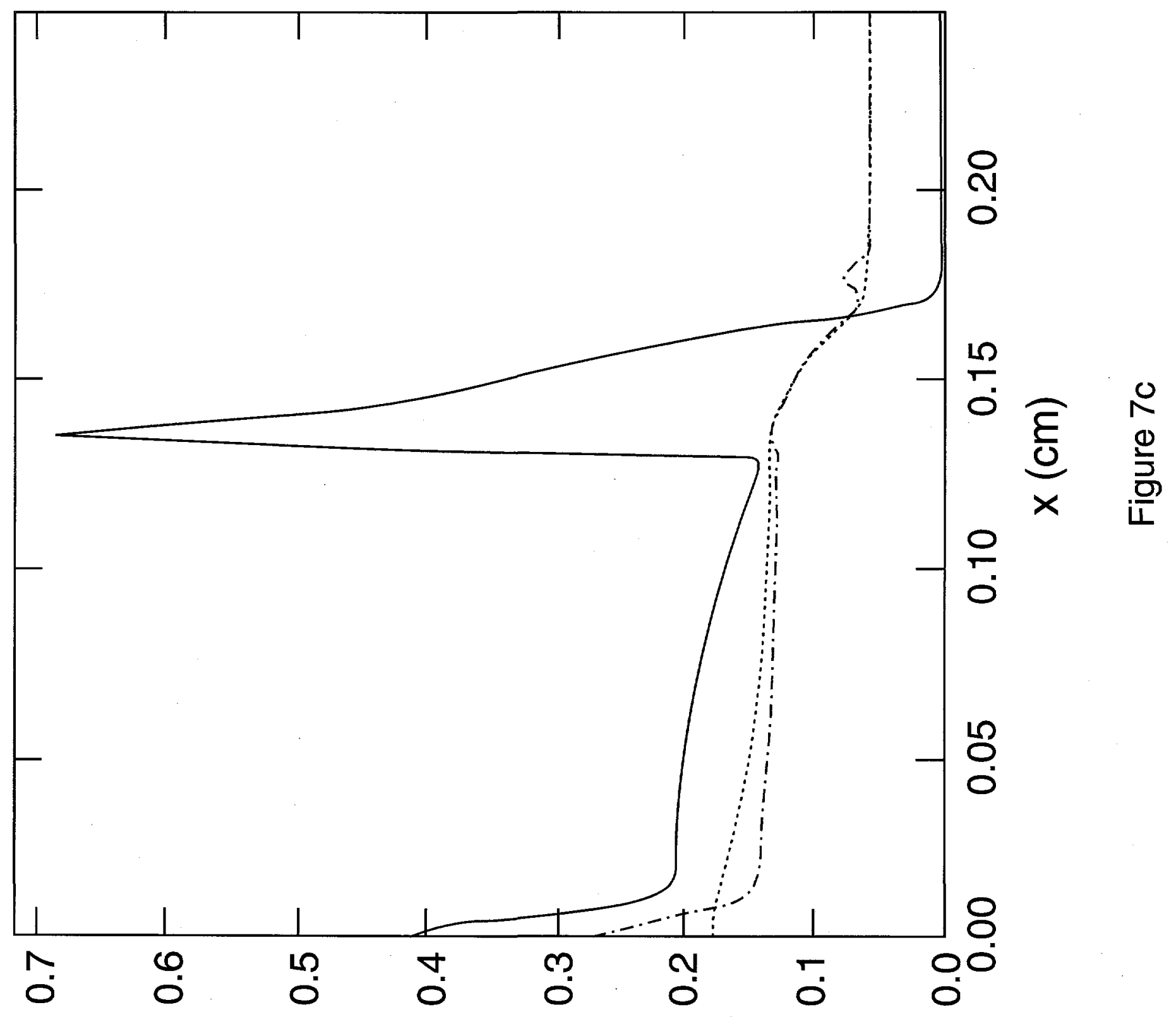




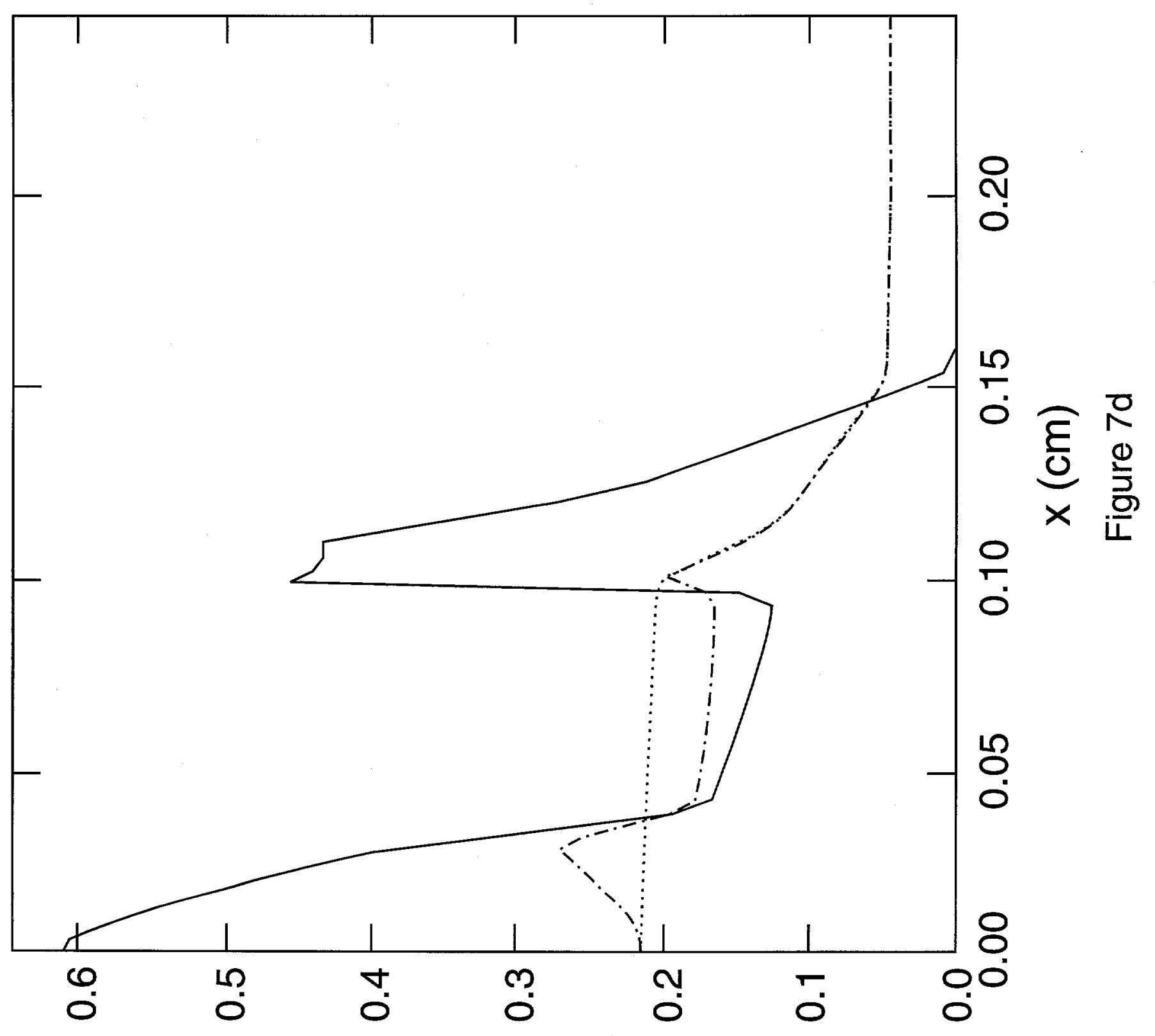




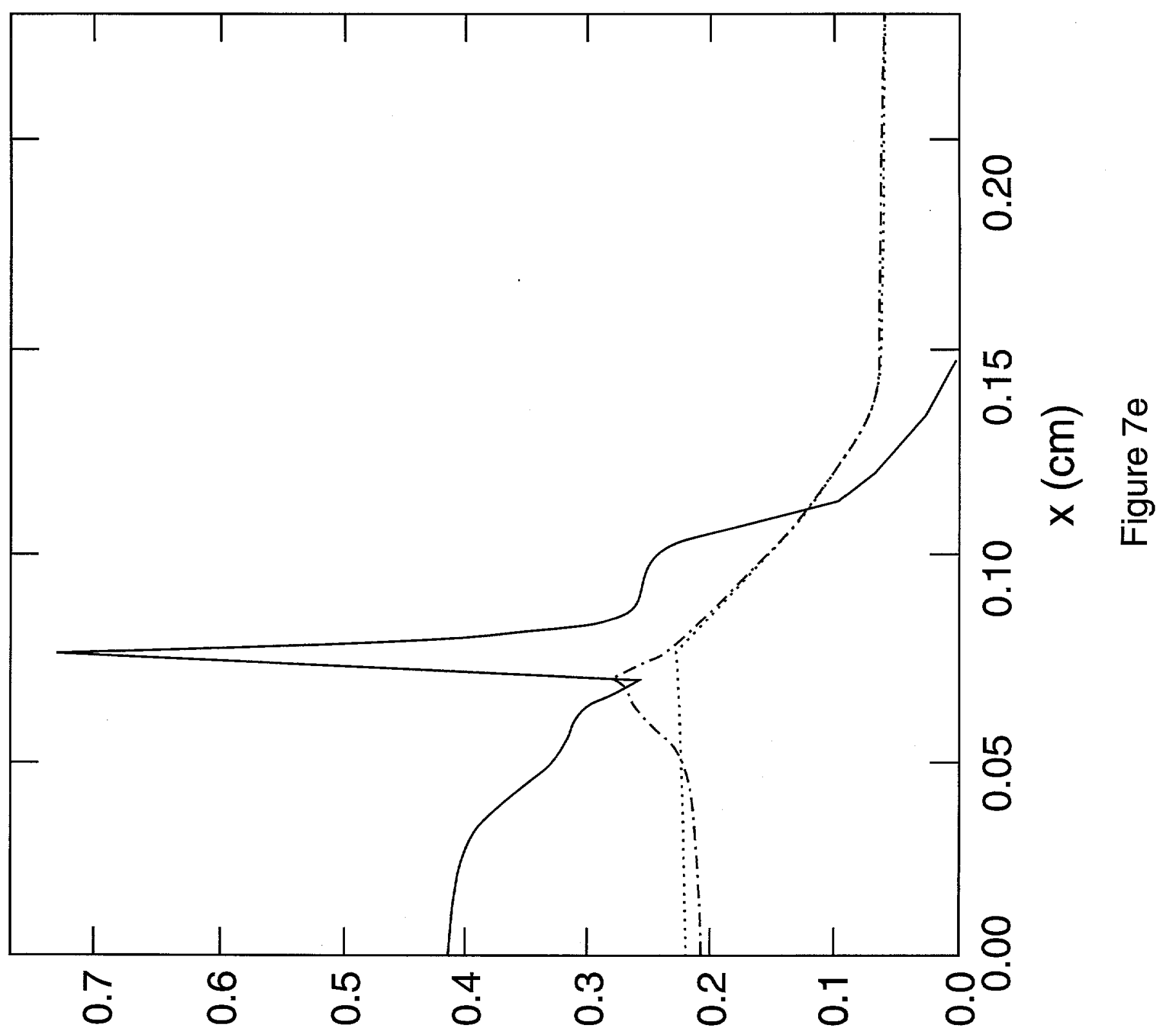




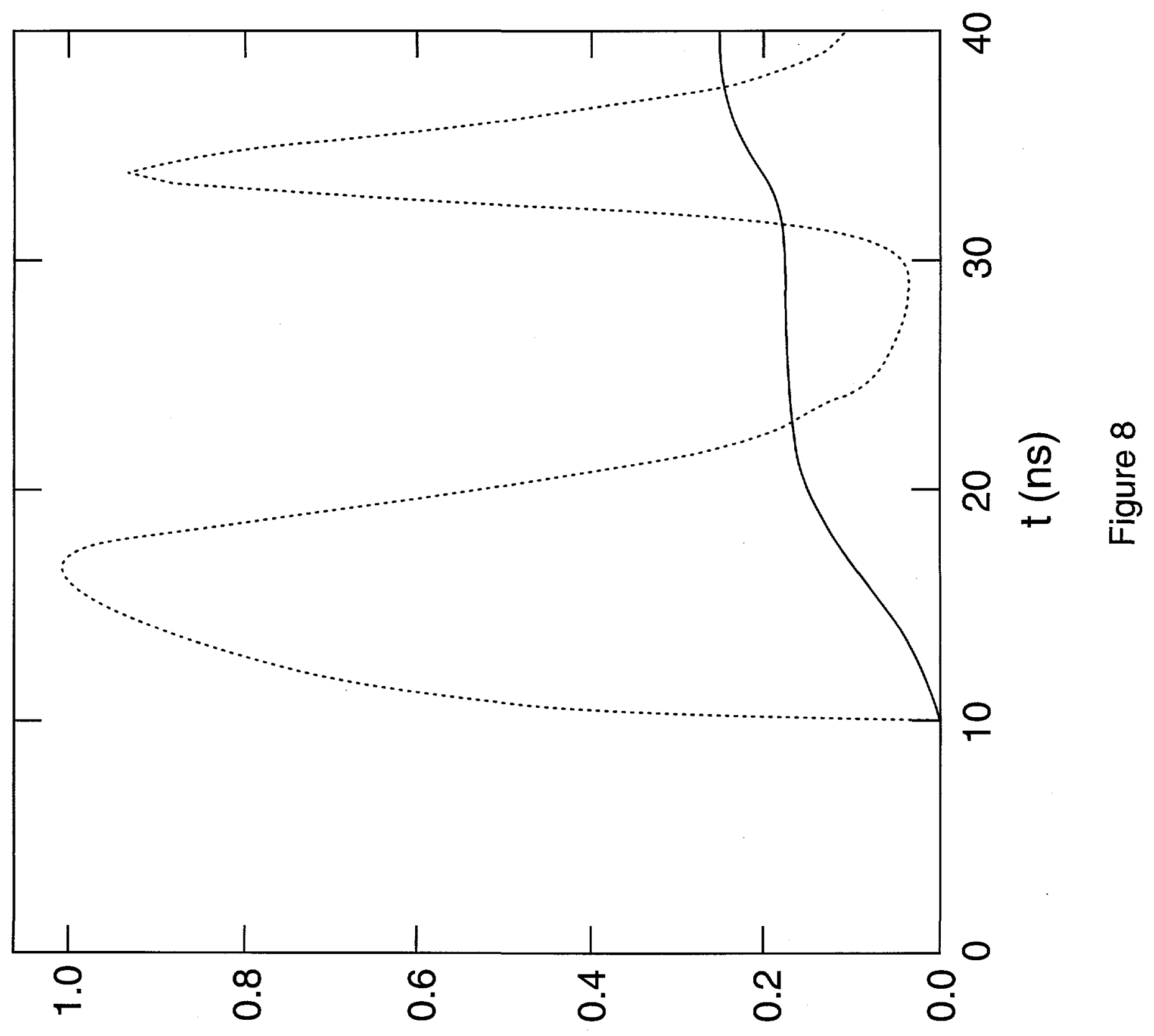




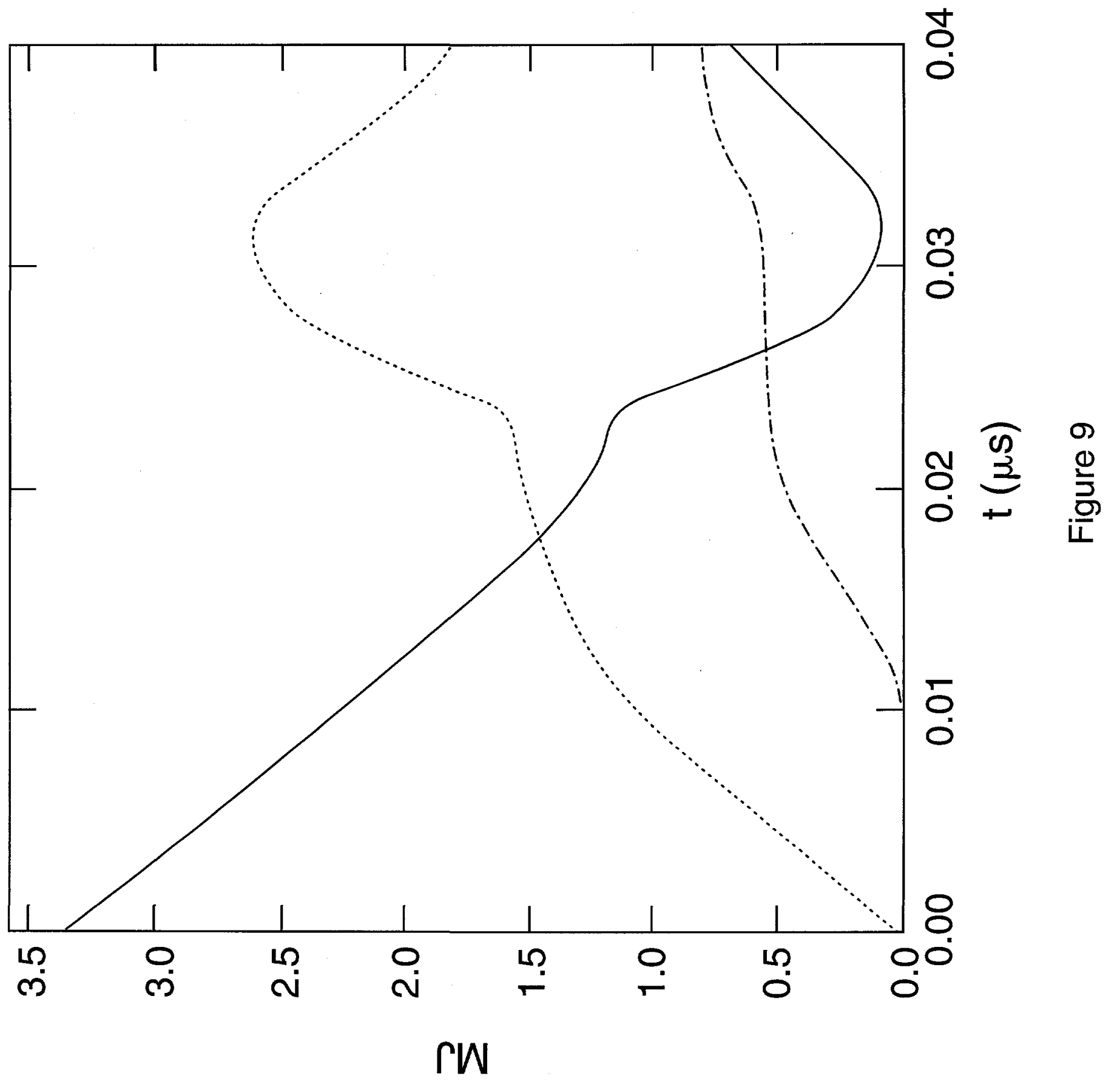




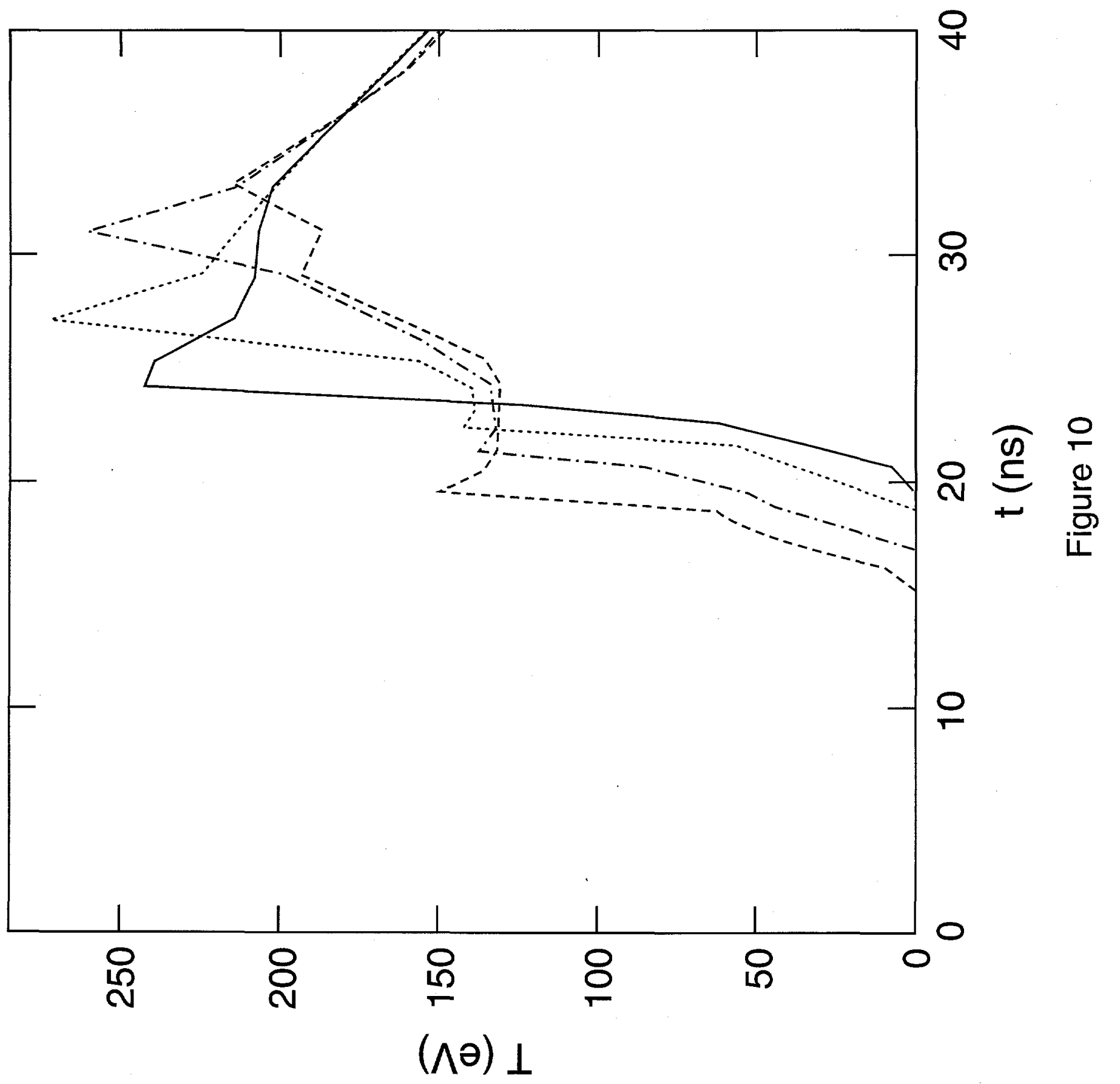




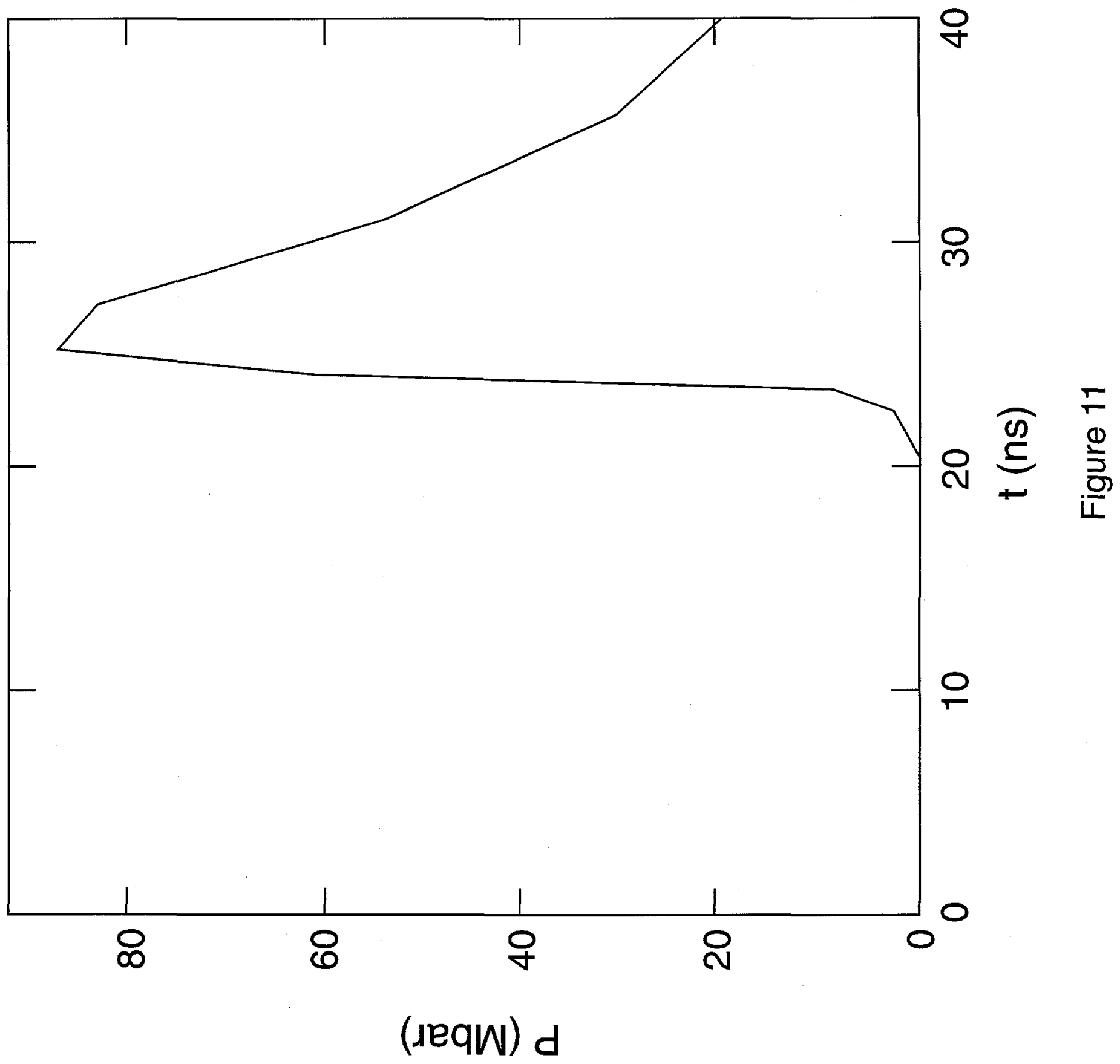

